\title{
Dirac operators and symmetries of quasitoric manifolds
}

\author{
MICHAEL WIEMELER
}

\begin{abstract}
We establish a vanishing result for indices of certain twisted Dirac operators on $\operatorname{Spin}^{c}$-manifolds with nonabelian Lie group actions. We apply this result to study nonabelian symmetries of quasitoric manifolds. We give upper bounds for the degree of symmetry of these manifolds.
\end{abstract}

$57 \mathrm{~S} 15,57 \mathrm{~S} 25,58 \mathrm{~J} 20$

\section{Introduction}

A quasitoric manifold is a $2 n$-dimensional manifold with a well-behaved action of an $n$-dimensional torus such that the orbit space is an $n$-dimensional simple polytope. Quasitoric manifolds were introduced by Davis and Januszkiewicz [6] as topological generalizations of nonsingular projective toric varieties.

In this paper we study the degree of symmetry of quasitoric manifolds and give upper bounds in various situations. For example we show that $\mathbb{C} \mathrm{P}^{n}$ is the most symmetric $2 n$-dimensional quasitoric manifold. Moreover, we construct infinitely many quasitoric manifolds of dimension $2 n=4 k, k>0$, that do not admit an action of a semisimple compact connected Lie group.

For a smooth manifold $M$, the degree of symmetry $N(M)$ of $M$ is defined to be the maximum of the dimensions of those compact Lie groups that act smoothly and effectively on $M$.

Similarly one defines the semisimple symmetry degree $N^{s s}(M)$ of $M$ as

$$
N^{s s}(M)=\max \left\{\begin{array}{l|l}
\operatorname{dim} G & \begin{array}{l}
G \text { compact semisimple Lie group, } \\
G \text { acts smoothly and effectively on } M
\end{array}
\end{array}\right\}
$$

and the torus symmetry degree $T(M)$ of $M$ to be the maximum of the dimensions of those compact tori that act smoothly and effectively on $M$.

It is well known that, for an $n$-dimensional manifold $M, N(M) \leq n(n+1) / 2$ with equality holding if and only if $M=S^{n}$ or $M=\mathbb{R P}{ }^{n}$. Moreover, we have $T(M) \leq n$ 
with equality holding if and only if $M$ is a torus. If $\chi(M) \neq 0$, then we have $T(M) \leq n / 2$.

A quasitoric manifold has positive Euler characteristic. Therefore the torus symmetry degree of a quasitoric manifold is maximal in the class of manifolds with nonvanishing Euler characteristic.

In this paper we show that $\mathbb{C P}^{n}$ has maximal degree of symmetry among the quasitoric manifolds of dimension $2 n$, ie $N(M)<N\left(\mathbb{C P}^{n}\right)=n^{2}+2 n$ for all quasitoric manifolds $M \neq \mathbb{C P}^{n}$ with $\operatorname{dim} M=2 n$ (see Theorem 8.1).

Moreover, we generalize a vanishing result for indices of certain twisted Dirac operators on $\operatorname{Spin}^{c}$-manifolds with Pin(2)-action found by Dessai [7] to manifolds with actions of more general groups (see Theorem 2.4). This generalization allows us to prove that if a $2 n$-dimensional $\operatorname{Spin}^{c}$-manifold $M$ with $\chi(M) \neq 0$ admits such a twisted Dirac operator with nonvanishing index then its degree of symmetry is bounded from above by $3 n$ with equality holding if and only if $M=\prod_{i=1}^{n} S^{2}$ (see Corollary 2.14). We show that a $2 n$-dimensional quasitoric manifold whose orbit polytope admits a facet coloring with $n$ colors is an example of such a manifold. Hence, we get:

Theorem 1.1 (Corollary 5.4) If $M$ is a $2 n$-dimensional quasitoric manifold whose orbit polytope admits a facet coloring with $n$ colors, then we have $N(M) \leq 3 n$ with equality holding if and only if $M=\prod_{i=1}^{n} S^{2}$.

Moreover, we show that if a $2 n$-dimensional $\operatorname{Spin}^{c}$-manifold $M$ admits a twisted Dirac operator with nonvanishing index and an effective action of a nonabelian compact connected Lie group $G$, then the order of the Weyl group of $G$ divides the Euler characteristic of $M$ (see Corollary 2.12). This enables us to prove the following result.

Theorem 1.2 (Corollary 5.6, Corollary 5.8) Let $n \geq 2$. Then we have:

(1) If $n$ is odd, then there are infinitely many quasitoric manifolds $M$ of dimension $2 n$ with $N^{s s}(M) \leq 3$, ie the only semisimple simply connected compact Lie group that can act almost effectively on $M$ is $\mathrm{SU}(2)$.

(2) If $n$ is even, then there are infinitely many quasitoric manifolds of dimension $2 n$ on which no semisimple compact connected Lie group can act effectively.

We also study those $2 n$-dimensional quasitoric manifolds whose orbit polytopes admit facet colorings with $n$ colors and have relatively many nonabelian symmetries. For these manifolds we have the following theorem. 
Theorem 1.3 (Theorem 6.2, Theorem 7.2) Let $M$ be a $2 n$-dimensional quasitoric manifold whose orbit polytope admits a facet coloring with $n$ colors. Assume that one of the following two conditions holds:

(1) There is an action of a compact Lie group on $M$ such that $\operatorname{dim} M / G \leq 1$.

(2) We have $N(M) \geq 3 n-4$.

Then $M$ is the total space of a fiber bundle over $\prod S^{2}$.

By considering twisted Dirac operators we can also prove the following theorem:

Theorem 1.4 (Corollary 4.6) Let $M$ be a Spin-manifold with $p_{1}(M)=0, G$ an exceptional Lie group or $G=\operatorname{Spin}(2 l+1)$ or $G=\operatorname{Sp}(l)$ with $l=1,3,6$ or $l \geq 15$ and $T$ a maximal torus of $G$. If the Witten genus of $M$ does not vanish, then we have $N^{s s}\left(M \times \prod_{i=1}^{k} G / T\right)=k \operatorname{dim} G$.

If more generally $G$ is a semisimple compact connected Lie group with maximal torus $T$, then we still get upper bounds for the semisimple symmetry degree of $M \times G / T$. But we do not get the exact value of $N^{s s}(M \times G / T)$ in the more general setting. It should be noted here, that it has been shown by Hauschild [11] that the semisimple symmetry degree of $G / T$ is equal to $\operatorname{dim} G$ if $G$ is a semisimple compact connected Lie group with maximal torus $T$. So Theorem 1.4 may be viewed as a partial generalization of his result.

This paper is organized as follows. In Sections 2 and 3 we discuss indices of twisted Dirac operators on $\mathrm{Spin}^{c}$-manifolds. Then we prove Theorem 1.4 in Section 4. In Section 5 we apply the results of the previous sections to show that there are quasitoric manifolds with low semisimple symmetry degree. In Sections 6 and 7 we study those quasitoric manifolds that have a nonvanishing index and have relatively many nonabelian symmetries. In Section 8 we show that $\mathbb{C P}^{n}$ is the most symmetric quasitoric manifold in dimension $2 n$. That section is independent of the other sections. In an appendix we prove some technical details that are needed in Section 2.

\section{Acknowledgements}

I would like to thank Anand Dessai for comments on an earlier version of this paper. I did the research for this paper while I was staying in Manchester. Therefore I would also like to thank Nigel Ray and the University of Manchester for hospitality. During my stay in Manchester I was supported by grant number PBFRP2-133466 from the Swiss National Science Foundation. 


\section{Twisted Dirac operators and nonabelian Lie group actions}

The purpose of this section is to generalize some results of Dessai [7] to a class of nonabelian compact nonconnected Lie groups.

We begin with a review of some well known facts about $\operatorname{Spin}^{c}$-manifolds and the results of Dessai $[7 ; 8]$. For more background information about the group $\operatorname{Spin}^{c}(k)$ and $\operatorname{Spin}^{c}$-structures on manifolds see for example Atiyah, Bott and Shapiro [1], Petrie [16] and Hattori [9].

An orientable manifold $M$ has a Spin ${ }^{c}$-structure if and only if the second StiefelWhitney class $w_{2}(M)$ of $M$ is the $\bmod 2-$ reduction of an integral class $c \in H^{2}(M ; \mathbb{Z})$. Associated to a $\operatorname{Spin}^{c}$-structure on $M$ there is a complex line bundle. We denote the first Chern class of this line bundle by $c_{1}^{c}(M)$. Its mod 2-reduction is $w_{2}(M)$ and we should note that any integral cohomology class whose mod 2-reduction is $w_{2}(M)$ may be realized as the first Chern class of a line bundle associated to some Spin $^{c}$-structure on $M$.

Let $M$ be a $2 n$-dimensional Spin ${ }^{c}$-manifold on which $S^{1}$ acts smoothly. We say that the $S^{1}$-action on $M$ lifts into the Spin ${ }^{c}$-structure $P$ if there is a $S^{1}$-action on $P$ that commutes with the $\operatorname{Spin}^{c}(2 n)$-action on $P$ such that the projection $P \rightarrow M$ is $S^{1}$-equivariant.

Lemma 2.1 The $S^{1}$-action on $M$ lifts into the $\operatorname{Spin}^{c}$-structure if and only if it lifts to an action on the line bundle associated to the $\operatorname{Spin}^{c}$-structure.

Proof If the $S^{1}$-action lifts to an action on the $\operatorname{Spin}^{c}$-structure $P$ of $M$, then it also lifts into the associated line bundle $P \times \operatorname{Spin}^{c} \mathbb{C}$.

Now assume that the $S^{1}$-action on $M$ lifts into the associated line bundle of $P$. Let $Q$ be the oriented orthogonal frame bundle of $M$. Then the $S^{1}$-action lifts into $Q$. Moreover, by Petrie [16, pages 127-128], the action on $M$ lifts into $P$ if and only if the action on $Q$ lifts into the $S^{1}$-bundle

$$
\xi: P \longrightarrow P / S^{1}=Q .
$$

Now we consider the Serre spectral sequence for the fibration $Q \rightarrow Q S^{1} \rightarrow B S^{1}$. By Hattori and Yoshida [10, Corollary 1.3], the $S^{1}$-action lifts into $\xi$ if and only if

$$
d_{2} c_{1}(\xi)=0 \quad \text { and } \quad d_{3} c_{1}(\xi)=0 .
$$

Because $H^{*}\left(B S^{1} ; \mathbb{Z}\right)$ is concentrated in even degrees, this holds if and only if $d_{2} c_{1}(\xi)=0$. 
Let $\xi^{\prime}$ be the $S^{1}$-bundle over $Q$ associated to the pullback of the line bundle associated to $P$. Then the $S^{1}$-action on $Q$ lifts into $\xi^{\prime}$. Since $\xi^{\prime}=\xi^{2}$, we have $2 d_{2} c_{1}(\xi)=$ $d_{2} c_{1}\left(\xi^{\prime}\right)=0$. Because $E_{2}^{2,1}=H^{2}\left(B S^{1} ; H^{1}(Q ; \mathbb{Z})\right)$ is torsion-free, it follows that the $S^{1}$-action lifts into $P$.

If the $S^{1}$-action on $M$ lifts into the $\operatorname{Spin}^{c}$-structure, then we have an $S^{1}$-equivariant Spin ${ }^{c}$-Dirac operator $\partial_{c}$. Its $S^{1}$-equivariant index is an element of the representation ring of $S^{1}$ and is defined as

$$
\operatorname{ind}_{S^{1}}\left(\partial_{c}\right)=\operatorname{ker} \partial_{c}-\operatorname{coker} \partial_{c} \in R\left(S^{1}\right) .
$$

Let $V$ be a $S^{1}$-equivariant complex vector bundle over $M$ and $W$ an even-dimensional $S^{1}$-equivariant Spin vector bundle over $M$. With this data we build a power series $R \in K_{S^{1}}(M) \llbracket q \rrbracket$ defined by

$$
\begin{aligned}
& R=\bigotimes_{k=1}^{\infty} S_{q^{k}}\left(\tilde{T M} \otimes_{\mathbb{R}} \mathbb{C}\right) \otimes \Lambda_{-1}\left(V^{*}\right) \otimes \bigotimes_{k=1}^{\infty} \Lambda_{-q^{k}}\left(\tilde{V} \otimes_{\mathbb{R}} \mathbb{C}\right) \\
& \otimes \Delta(W) \otimes \bigotimes_{k=1}^{\infty} \Lambda_{q^{n}}\left(\tilde{W} \otimes_{\mathbb{R}} \mathbb{C}\right) .
\end{aligned}
$$

Here $q$ is a formal variable, $\widetilde{E}$ denotes the reduced vector bundle $E-\operatorname{dim} E, \Delta(W)$ is the full complex spinor bundle associated to the Spin-vector bundle $W$, and $\Lambda_{t}$ (respectively $S_{t}$ ) denotes the exterior (respectively symmetric) power operation. The tensor products are, if not indicated otherwise, taken over the complex numbers.

After extending ind $S^{1}$ to power series we may define:

Definition 2.2 Let $\varphi^{c}(M ; V, W)_{S^{1}}$ be the $S^{1}$-equivariant index of the $\operatorname{Spin}^{c}$-Dirac operator twisted with $R$ :

$$
\varphi^{c}(M ; V, W)_{S^{1}}=\operatorname{ind}_{S^{1}}\left(\partial_{c} \otimes R\right) \in R\left(S^{1}\right) \llbracket q \rrbracket .
$$

We denote by $\varphi^{c}(M ; V, W)$ the nonequivariant version of this index:

$$
\varphi^{c}(M ; V, W)=\operatorname{ind}\left(\partial_{c} \otimes R\right) \in \mathbb{Z} \llbracket q \rrbracket .
$$

The Atiyah-Singer index theorem [2] allows us to calculate

$$
\varphi^{c}(M ; V, W)=\left\langle e^{c_{1}^{c}(M) / 2} \operatorname{ch}(R) \hat{A}(M),[M]\right\rangle .
$$

Here we have

$$
\operatorname{ch}(R)=Q_{1}(T M) Q_{2}(V) Q_{3}(W),
$$


with

$$
\begin{aligned}
Q_{1}(T M) & =\operatorname{ch}\left(\bigotimes_{k=1}^{\infty} S_{q^{k}}\left(\widetilde{T M} \otimes_{\mathbb{R}} \mathbb{C}\right)\right)=\prod_{i} \prod_{k=1}^{\infty} \frac{\left(1-q^{k}\right)^{2}}{\left(1-e^{x_{i}} q^{k}\right)\left(1-e^{-x_{i}} q^{k}\right)} \\
Q_{2}(V) & =\operatorname{ch}\left(\Lambda_{-1}\left(V^{*}\right) \otimes \bigotimes_{k=1}^{\infty} \Lambda_{-q^{k}}\left(\tilde{V} \otimes_{\mathbb{R}} \mathbb{C}\right)\right) \\
& =\prod_{i}\left(1-e^{-v_{i}}\right) \prod_{k=1}^{\infty} \frac{\left(1-e^{v_{i}} q^{k}\right)\left(1-e^{-v_{i}} q^{k}\right)}{\left(1-q^{k}\right)^{2}} \\
Q_{3}(W) & =\operatorname{ch}\left(\Delta(W) \otimes \bigotimes_{k=1}^{\infty} \Lambda_{q^{n}}\left(\tilde{W} \otimes_{\mathbb{R}} \mathbb{C}\right)\right) \\
& =\prod_{i}\left(e^{w_{i} / 2}+e^{-w_{i} / 2}\right) \prod_{k=1}^{\infty} \frac{\left(1+e^{w_{i}} q^{k}\right)\left(1+e^{-w_{i}} q^{k}\right)}{\left(1+q^{k}\right)^{2}}
\end{aligned}
$$

Here $\pm x_{i}$ (respectively $v_{i}$ and $\pm w_{i}$ ) denote the formal roots of $T M$ (respectively $V$ and $W$ ). If $c_{1}^{c}(M)=c_{1}(V)$, then we have

$$
e^{c_{1}^{c}(M) / 2} Q_{2}(V)=e(V) \frac{1}{\widehat{A}(V)} \prod_{i} \prod_{k=1}^{\infty} \frac{\left(1-e^{v_{i}} q^{k}\right)\left(1-e^{-v_{i}} q^{k}\right)}{\left(1-q^{k}\right)^{2}}=e(V) Q_{2}^{\prime}(V) .
$$

Note that if $M$ is a Spin-manifold, then there is a canonical $\operatorname{Spin}^{c}$-structure on $M$. With this $\operatorname{Spin}^{c}$-structure $\varphi^{c}(M ; 0, T M)$ is equal to the elliptic genus of $M$. Moreover, $\varphi^{c}(M ; 0,0)$ is the Witten genus of $M$.

Dessai [8, Theorem 3.2] proved the following:

Theorem 2.3 Assume that the equivariant Pontrjagin class $p_{1}^{S^{1}}(V+W-T M)$ restricted to $M^{S^{1}}$ is equal to $\pi_{S^{1}}^{*}\left(I x^{2}\right)$ modulo torsion, where $\pi S^{1}: B S^{1} \times M^{S^{1}} \rightarrow B S^{1}$ is the projection on the first factor, $x \in H^{2}\left(B S^{1} ; \mathbb{Z}\right)$ is a generator and $I$ is an integer. Assume, moreover, that $c_{1}^{c}(M)$ and $c_{1}(V)$ are equal modulo torsion. If $I<0$, then $\varphi^{c}(M ; V, W)_{S^{1}}$ vanishes identically.

Let $G$ be a compact Lie group such that:

(1) There is an exact sequence of Lie groups

$$
1 \longrightarrow T \longrightarrow G \longrightarrow W(G) \longrightarrow 1,
$$

where $T$ is a torus and $W(G)$ a finite group. 
(2) If condition (1) holds, then $G$ acts by conjugation on $T$. Since $T$ is abelian this action factors through $W(G)$. We assume that this action of $W(G)$ is nontrivial on $T$.

An action of $G$ on a manifold $M$ is called nice if $G$ acts almost effectively on $M$ and if the induced action on $H^{*}(M ; \mathbb{Z})$ is trivial.

For nice $G$-actions on $\operatorname{Spin}^{c}$-manifolds we have the following vanishing result.

Theorem 2.4 Let $M$ be a Spin ${ }^{c}$-manifold on which $G$ acts nicely such that $M^{G} \neq \varnothing$. Let $V$ and $W$ be sums of complex line bundles over $M$ such that $W$ is Spin, $c_{1}(V)=c_{1}^{c}(M)$ modulo torsion and $p_{1}(V+W-T M)=0$ modulo torsion. Assume that $b_{1}(M)=0$ or that the $G$-action on $M$ extends to an action of a simply connected compact Lie group. Then $\varphi^{c}(M ; V, W)$ vanishes.

Remark 2.5 Theorem 2.4 is a generalization of [7, Theorem 4.4].

Before we prove Theorem 2.4 we state three lemmas about the equivariant cohomology of $G$-manifolds that are needed in the proof.

Lemma 2.6 Let $M$ be a nice $G$-manifold such that $M^{G} \neq \varnothing$ and $b_{1}(M)=0$. Then

$$
0 \longrightarrow H^{2}(B G ; \mathbb{Z}) \longrightarrow H_{G}^{2}(M ; \mathbb{Z}) \longrightarrow H^{2}(M ; \mathbb{Z}) \longrightarrow 0
$$

is exact.

Proof We consider the Serre spectral sequence for the fibration $M \rightarrow M_{G} \rightarrow B G$. Because the $G$-action on $M$ is nice we have

$$
E_{2}^{p, q}=H^{p}\left(B G ; H^{q}(M ; \mathbb{Z})\right) .
$$

Since $b_{1}(M)=0$, we have $E_{\infty}^{1,1}=0$. Therefore we have an exact sequence

$$
0 \longrightarrow E_{\infty}^{2,0} \longrightarrow H_{G}^{2}(M ; \mathbb{Z}) \longrightarrow E_{\infty}^{0,2} \longrightarrow 0 .
$$

Because $M^{G} \neq \varnothing, H^{*}(B G ; \mathbb{Z}) \rightarrow H_{G}^{*}(M ; \mathbb{Z})$ is injective. Hence, we have

$$
H^{*}(B G ; \mathbb{Z})=E_{2}^{*, 0}=E_{\infty}^{*, 0}
$$

and the differentials $d_{r}: E_{r}^{*-r, r-1} \rightarrow E_{r}^{*, 0}$ vanish.

It remains to show that $E_{\infty}^{0,2}=E_{2}^{0,2}=H^{2}(M ; \mathbb{Z})$. That is equivalent to $d_{r}: E_{r}^{0,2} \rightarrow$ $E_{r}^{r, 3-r}$ vanishing for all $r$.

Now we have $E_{2}^{2,1}=0$ because $b_{1}(M)=0$. Therefore $d_{2}$ vanishes. Since there are $G$-fixed points in $M, d_{3}$ vanishes. The differentials $d_{r}, r>3$, vanish because $E_{r}^{r, 3-r}=0$ for $r>3$. Therefore the statement follows. 
Lemma 2.7 Let $M$ be a nice $G$-manifold. If the $G$-action on $M$ extends to an action of a simply connected compact Lie group $\widehat{G}$, then the natural map

$$
H_{\widehat{G}}^{2}(M ; \mathbb{Z}) \longrightarrow H^{2}(M ; \mathbb{Z})
$$

is an isomorphism. Moreover, $H_{G}^{2}(M ; \mathbb{Z}) \rightarrow H^{2}(M ; \mathbb{Z})$ is surjective.

Proof Since $B \widehat{G}$ is three-connected the first statement follows from an inspection of the Serre spectral sequence for the fibration $M \rightarrow M_{\widehat{G}} \rightarrow B \widehat{G}$ as in the proof of Lemma 2.6. Then the second statement follows because $H_{\widehat{G}}^{2}(M ; \mathbb{Z}) \rightarrow H^{2}(M ; \mathbb{Z})$ factors through $H_{G}^{2}(M ; \mathbb{Z})$.

Lemma 2.8 Assume that $T^{W(G)}$ is finite or equivalently that $\operatorname{dim}(L T)^{W(G)}=0$ or $\operatorname{dim}\left(L T^{*}\right)^{W(G)}=0$. Let $M$ be a nice $G$-manifold, then

$$
H^{4}(B G ; \mathbb{Q}) \longrightarrow H_{G}^{4}(M ; \mathbb{Q}) \longrightarrow H^{4}(M ; \mathbb{Q})
$$

is exact.

Proof Because $\operatorname{dim}\left(L T^{*}\right)^{W(G)}=0$, we have by Borel [3, Proposition 20.4]

$$
H^{i}(B G ; \mathbb{Q})=0
$$

for $i=1,2,3$. Therefore from the Serre spectral sequence of the fibration $M \rightarrow$ $M_{G} \rightarrow B G$ we get an exact sequence

$$
0 \longrightarrow E_{\infty}^{4,0} \longrightarrow H_{G}^{4}(M ; \mathbb{Q}) \longrightarrow E_{\infty}^{0,4} \longrightarrow 0 .
$$

Since $H^{4}(B G ; \mathbb{Q})$ surjects to $E_{\infty}^{4,0}$ and $E_{\infty}^{0,4}$ injects into $H^{4}(M ; \mathbb{Q})$, the statement follows.

Now we are ready to prove Theorem 2.4 in two special cases. The general case will follow from these special cases.

Lemma 2.9 Assume that $T^{W(G)}$ is finite. Then Theorem 2.4 holds.

Proof Let $V=\bigoplus L_{i}$ and $W=\bigoplus L_{i}^{\prime}$ with $L_{i}, L_{i}^{\prime}$ line bundles. By Lemmas 2.6, 2.7 and [10, Corollary 1.2], the $G$-action on $M$ lifts into each line bundle $L_{i}, L_{i}^{\prime}$. Therefore $p_{1}^{G}(V+W-T M)$ is well defined. Moreover, by Lemma 2.1, the action of every $S^{1} \subset T \subset G$ lifts into the $\operatorname{Spin}^{c}$-structure on $M$.

By Theorem 2.3, it is sufficient to show that, for $S^{1} \hookrightarrow T \hookrightarrow G$,

$$
p_{1}^{S^{1}}(V+W-T M)=\rho\left(S^{1}, G\right)^{*} p_{1}^{G}(V+W-T M)=a \pi_{S^{1}}^{*}\left(x^{2}\right),
$$


with $a \in \mathbb{Q}, a<0$, and $x \in H^{2}\left(B S^{1} ; \mathbb{Z}\right)$ a generator. Here $\rho\left(S^{1}, G\right)^{*}: H_{G}^{*}(M ; \mathbb{Q}) \rightarrow$ $H_{S^{1}}^{*}(M ; \mathbb{Q})$ is the map induced by the inclusion $S^{1} \hookrightarrow G$ and $\pi_{S^{1}}^{*}: H^{*}\left(B S^{1} ; \mathbb{Q}\right) \rightarrow$ $H_{S^{1}}^{*}(M ; \mathbb{Q})$ is the natural map.

By Lemma 2.8, there is an $\alpha \in H^{4}(B G ; \mathbb{Q})$ with $\pi_{G}^{*}(\alpha)=p_{1}^{G}(V+W-T M)$. Therefore we have

$$
p_{1}^{S^{1}}(V+W-T M)=\pi_{S^{1}}^{*} \rho\left(S^{1}, G\right)^{*} \alpha=a \pi_{S^{1}}^{*}\left(x^{2}\right)
$$

with $a \in \mathbb{Q}$. It remains to show that $a<0$. We restrict $p_{1}^{S^{1}}(V+W-T M)$ to a $G$-fixed point $y$. Then we have

$$
\left.p_{1}^{S^{1}}(V+W-T M)\right|_{y}=\sum \alpha_{i}^{2}+\sum \beta_{i}^{2}-\sum \gamma_{i}^{2},
$$

where $\alpha_{i}$ is the weight of the $S^{1}$-representation on the fiber of $L_{i}$ over $y, \beta_{i}$ is the weight of the $S^{1}$-representation on the fiber of $L_{i}^{\prime}$ over $y$ and the $\gamma_{i}$ are the weights of the $S^{1}$-representation $T_{y} M$.

The representations on the fibers of $L_{i}, L_{i}^{\prime}$ are restrictions of one-dimensional $G$ representations to $S^{1}$. Because $\left(L T^{*}\right)^{W(G)}=0$, all such representations are trivial. Therefore $a=-\sum \gamma_{i}^{2}<0$ follows, because $S^{1}$ acts nontrivially on $M$.

Lemma 2.10 Assume that $W(G)$ is cyclic. Then Theorem 2.4 holds.

Proof We show that $G$ has a subgroup satisfying the assumptions of Lemma 2.9. Then the statement follows from that lemma.

By Lemma A.2, there are two $W(G)$-invariant subtori $T_{1}$ and $T_{2}$ of $T$ such that:

- $W(G)$ acts trivially on $T_{1}$.

- $T_{2}^{W(G)}$ is finite.

- $\quad T$ is generated by $T_{1}$ and $T_{2}$.

Let $g \in G$ be a preimage of a generator of $W(G)$. Then we have $g^{\# W(G)} \in T$. Let $t_{1} \in T_{1}$ and $t_{2} \in T_{2}$ such that $g^{\# W(G)}=t_{1} t_{2}$. Moreover, let $t \in T_{1}$ such that $t_{1}=t^{\# W(G)}$. Then $g t^{-1}$ is another preimage of the generator of $W(G)$ and $\left(g t^{-1}\right)^{\# W(G)} \in T_{2}$.

Let $G^{\prime}$ be the subgroup of $G$ generated by $g t^{-1}$ and $T_{2}$. Then there is an exact sequence

$$
1 \longrightarrow T_{2} \longrightarrow G^{\prime} \longrightarrow W(G) \longrightarrow 1
$$

Therefore $G^{\prime}$ satisfies the assumptions of Lemma 2.9. 
In the situation of Theorem 2.4, there is always a cyclic subgroup $W(H)$ of $W(G)$ which acts nontrivially on $T$. If $H$ is the preimage of $W(H)$ under the map $G \rightarrow$ $W(G)$, then Theorem 2.4 follows from Lemma 2.10 applied to the restricted action of $H$ on $M$.

From Theorem 2.4 we get the following corollaries about actions of compact connected nonabelian Lie groups on $\mathrm{Spin}^{c}$-manifolds.

Corollary 2.11 Let $G$ be a compact connected nonabelian Lie group and $M$ a $\operatorname{Spin}^{c}$ manifold with $\varphi^{c}(M ; V, W) \neq 0$ and $V, W$ as in Theorem 2.4. Assume that $G$ acts almost effectively on $M$ and that $T$ is a maximal torus of $G$. Then, for all $x \in M^{T}$, $G_{x}=T$ holds.

Proof Let $\widetilde{G}=G^{\prime} \times T_{0}$ be a covering group of $G$ with $G^{\prime}$ a semisimple simply connected compact Lie group and $T_{0}$ a torus. Then we have $\widetilde{G}_{x}=G_{x}^{\prime} \times T_{0}$. We will show that $G_{x}^{\prime}$ is a maximal torus of $G^{\prime}$. From this the statement follows. Since, for each compact connected nonabelian Lie group $H$, there is a group homomorphism $\operatorname{Pin}(2) \rightarrow H$ with finite kernel, $G_{x}^{\prime 0}=T^{\prime}$ is a maximal torus of $G^{\prime}$ by Theorem 2.4.

Assume that $G_{x}^{\prime} \neq T^{\prime}$. Then there is an exact sequence

$$
1 \longrightarrow T^{\prime} \longrightarrow G_{x}^{\prime} \longrightarrow G_{x}^{\prime} / T^{\prime} \longrightarrow 1
$$

and we have $G_{x}^{\prime} / T^{\prime} \subset N_{G^{\prime}} T^{\prime} / T^{\prime}$. Therefore $G_{x}^{\prime} / T^{\prime}$ acts nontrivially on $T^{\prime}$. But this is a contradiction to Theorem 2.4.

Corollary 2.12 Let $M$ and $G$ as in Corollary 2.11. Then $\# W(G) \mid \chi(M)$.

Proof We have $\chi(M)=\chi\left(M^{T}\right)$, where $T$ is a maximal torus of $G$. By Corollary 2.11, $W(G)$ acts freely on $M^{T}$. Therefore we get

$$
\chi(M)=\# W(G) \cdot \chi\left(M^{T} / W(G)\right) .
$$

The following two corollaries give upper bounds for the degree of symmetry of a $\operatorname{Spin}^{c}$-manifold which admits a twisted Dirac operator with nonzero index.

Corollary 2.13 Let $M$ be a $2 n$-dimensional $\operatorname{Spin}^{c}$-manifold with $\varphi^{c}(M ; V, W) \neq 0$ and $V, W$ as in Theorem 2.4 and $G$ be a compact connected Lie group with

(1) $\operatorname{dim} G-\operatorname{rank} G>2 n$, or

(2) $\operatorname{dim} G-\operatorname{rank} G=2 n$ and $\operatorname{rank} G<T(M)$.

Then there is no effective action of $G$ on $M$. 
Proof Let $\widetilde{G}=G^{\prime} \times T_{0}$ be a covering group of $G$ with $G^{\prime}$ a semisimple simply connected compact Lie group and $T_{0}$ a torus. Let $x \in M$. Then by Theorem 2.4 the identity component of $G_{x}^{\prime}$ must be a torus. Therefore $\operatorname{dim} G_{x} \leq \operatorname{rank} G$. Moreover, there is an embedding of $G / G_{x}$ in $M$. In case (1) this is impossible.

In case (2) we have, by dimension reasons, that $M=G / H$ and $H$ has maximal rank in $G$. By Corollary 2.11, $H$ must be a maximal torus of $G$. Moreover, $G$ is semisimple because it acts effectively on $M$. The torus symmetry degree of $G / H$ was calculated by Hauschild [11, Theorem 3.3]. It is equal to rank $G$, which contradicts our assumption that $\operatorname{rank} G<T(M)$.

Note that, if $G$ is a compact Lie group which acts effectively on a manifold $M$ as in the above corollary, then the rank of $G$ is bounded from above by the torus symmetry degree of $M$. Therefore we have $N(M) \leq 2 n+T(M)$. If the Euler characteristic of $M$ is nonzero, we have $T(M) \leq n$, so that we get the following corollary.

Corollary 2.14 Let $M$ be a $2 n$-dimensional $\operatorname{Spin}^{c}$-manifold with $\chi(M) \neq 0$ and $\varphi^{c}(M ; V, W) \neq 0$ with $V, W$ as in Theorem 2.4 and $G$ a compact connected Lie group which acts effectively on $M$. Then $\operatorname{dim} G \leq 3 n$. If $\operatorname{dim} G=3 n$, then $M=\prod S^{2}$.

Proof By the discussion above, we only have to prove the second statement.

If $\operatorname{dim} G=3 n$, then we must have $\operatorname{rank} G=n$ and $M=G / T$, where $T$ is a maximal torus of $G$. Therefore $G$ is semisimple. Because for a simple Lie group $G^{\prime}$ we have $\operatorname{dim} G^{\prime} \geq 3 \operatorname{rank} G^{\prime}$ with equality holding if and only if $G^{\prime}$ is a quotient of $\operatorname{SU}(2)$, we see that $G$ has a covering group of the form $\prod \mathrm{SU}(2)$. Therefore the statement follows.

\section{Products and connected sums}

In this section we discuss the calculation of the indices $\varphi^{c}(M ; V, W)$ for the case where $M$ is a connected sum or a product of $\operatorname{Spin}^{c}$-manifolds. The formulas derived here will be used in our applications of the results of the previous section in Sections 4 and 5 .

For cartesian products of $\operatorname{Spin}^{c}$-manifolds we have the following lemma.

Lemma 3.1 Let $M_{1}, M_{2}$ be even-dimensional Spin ${ }^{c}$-manifolds, $V_{i} \rightarrow M_{i}$ complex vector bundles and $W_{i} \rightarrow M_{i}, i=1,2$, Spin vector bundles. Then $M_{1} \times M_{2}$ is naturally a $\operatorname{Spin}^{c}$-manifold and

$$
\varphi^{c}\left(M_{1} \times M_{2} ; p_{1}^{*} V_{1} \oplus p_{2}^{*} V_{2}, p_{1}^{*} W_{1} \oplus p_{2}^{*} W_{2}\right)=\varphi^{c}\left(M_{1} ; V_{1}, W_{1}\right) \varphi^{c}\left(M_{2} ; V_{2}, W_{2}\right),
$$


where $p_{i}: M_{1} \times M_{2} \rightarrow M_{i}, i=1,2$, are the projections.

Proof Let $Q_{i} \in H^{\operatorname{dim} M_{i}}\left(M_{i} ; \mathbb{Q}\right) \llbracket q \rrbracket$ be the degree $\operatorname{dim} M_{i}$ part of

$$
e^{c_{1}^{c}\left(M_{i}\right) / 2} \operatorname{ch}(R) \hat{A}\left(M_{i}\right) \in H^{*}\left(M_{i} ; \mathbb{Q}\right) \llbracket q \rrbracket,
$$

$i=1,2$. Then we have

$$
\begin{aligned}
& \varphi^{c}\left(M_{1} \times M_{2} ; p_{1}^{*} V_{1} \oplus p_{2}^{*} V_{2}, p_{1}^{*} W_{1} \oplus p_{2}^{*} W_{2}\right) \\
&=\left\langle p_{1}^{*} Q_{1} p_{2}^{*} Q_{2},\left[M_{1} \times M_{2}\right]\right\rangle \\
&=\left\langle Q_{1},\left[M_{1}\right]\right\rangle\left\langle Q_{2},\left[M_{2}\right]\right\rangle \\
&=\varphi^{c}\left(M_{1} ; V_{1}, W_{1}\right) \varphi^{c}\left(M_{2} ; V_{2}, W_{2}\right) .
\end{aligned}
$$

The connected sum of two $\operatorname{Spin}^{c}$-manifolds is again a $\operatorname{Spin}^{c}$-manifold. For these manifolds we have the following lemma.

Lemma 3.2 Let $M_{1}, M_{2}$ be $\operatorname{Spin}^{c}$-manifolds of the same even dimension greater or equal to four, $V_{i} \rightarrow M_{i}, i=1,2$, complex vector bundles which are sums of complex line bundles and $W_{i} \rightarrow M_{i}, i=1,2$, Spin-bundles which are sums of complex line bundles such that

$$
c_{1}\left(V_{i}\right)=c_{1}^{c}\left(M_{i}\right) \quad \text { and } \quad p_{1}\left(V_{i}+W_{i}-T M_{i}\right)=0 .
$$

Then $M_{1} \# M_{2}$ has a Spin ${ }^{c}-$ structure, such that $c_{1}^{c}\left(M_{1} \# M_{2}\right)=c_{1}^{c}\left(M_{1}\right)+c_{1}^{c}\left(M_{2}\right)$. If $\operatorname{dim} V_{1}>\operatorname{dim} V_{2}$, then there are vector bundles $V \rightarrow M_{1} \# M_{2}, W \rightarrow M_{1} \# M_{2}$ which are sums of complex line bundles satisfying (3-1) such that

$$
\varphi^{c}\left(M_{1} \# M_{2} ; V, W\right)=2^{\operatorname{dim}_{\mathbb{C}} W_{2}} \varphi^{c}\left(M_{1} ; V_{1}, W_{1}\right) .
$$

If $\operatorname{dim} V_{1}=\operatorname{dim} V_{2}$, then the same holds with

$$
\varphi^{c}\left(M_{1} \# M_{2} ; V, W\right)=2^{\operatorname{dim}_{\mathbb{C}} W_{2}} \varphi^{c}\left(M_{1} ; V_{1}, W_{1}\right)+2^{\operatorname{dim}_{\mathbb{C}} W_{1}} \varphi^{c}\left(M_{2} ; V_{2}, W_{2}\right) .
$$

Proof Let $V_{i}=\bigoplus_{j=1}^{k_{i}} L_{j i}$ and $W_{i}=\bigoplus_{j=1}^{k_{i}^{\prime}} L_{j i}^{\prime}$ for $i=1,2$. Then the $L_{j i}, L_{j i}^{\prime}$ extend uniquely to vector bundles over $M_{1} \# M_{2}$, such that the restriction to $M_{k}, k \neq i$ is trivial. We denote these extensions also by $L_{j i}, L_{j i}^{\prime}$.

Let

$$
V=\bigoplus_{j=1}^{\max \left\{k_{1}, k_{2}\right\}} L_{j 1} \otimes L_{j 2} \quad \text { and } \quad W=\bigoplus_{i=1}^{2} \bigoplus_{j=1}^{k_{i}} L_{j i}^{\prime},
$$

where $L_{j i}$ is the trivial complex line bundle for $j>k_{i}$. 
The cohomology ring of $M_{1} \# M_{2}$ with coefficients in a ring $R$ is isomorphic to

$$
H^{*}\left(M_{1} ; R\right) \times H^{*}\left(M_{2} ; R\right) / I,
$$

where $I$ is the ideal generated by $(1,-1)$ and $\left(\xi_{1},-\xi_{2}\right)$. Here $\xi_{i}$ denotes the orientation class of $M_{i}$. Moreover, for the characteristic classes of $M_{1} \# M_{2}$ we have

$$
\begin{aligned}
& w_{i}\left(M_{1} \# M_{2}\right)=w_{i}\left(M_{1}\right)+w_{i}\left(M_{2}\right), \\
& p_{i}\left(M_{1} \# M_{2}\right)=p_{i}\left(M_{1}\right)+p_{i}\left(M_{2}\right), \quad \text { for } i>0 .
\end{aligned}
$$

Therefore there is a $\operatorname{Spin}^{c}$-structure on $M_{1} \# M_{2}$ with $c_{1}^{c}\left(M_{1} \# M_{2}\right)=c_{1}^{c}\left(M_{1}\right)+c_{1}^{c}\left(M_{2}\right)$.

For the vector bundles $V$ and $W$ defined above, we have

$$
\begin{aligned}
& c_{1}(V)=c_{1}\left(V_{1}\right)+c_{1}\left(V_{2}\right)=c_{1}^{c}\left(M_{1}\right)+c_{1}^{c}\left(M_{2}\right)=c_{1}^{c}\left(M_{1} \# M_{2}\right), \\
& p_{1}(V)=\sum_{j=1}^{k_{1}} c_{1}\left(L_{j 1}\right)^{2}+\sum_{j=1}^{k_{2}} c_{1}\left(L_{j 2}\right)^{2}=p_{1}\left(V_{1}\right)+p_{1}\left(V_{2}\right), \\
& p_{1}(W)=p_{1}\left(W_{1}\right)+p_{1}\left(W_{2}\right) .
\end{aligned}
$$

Therefore we have $p_{1}\left(V+W-T M_{1} \# M_{2}\right)=0$.

Now we have, assuming $\operatorname{dim} V_{1} \geq \operatorname{dim} V_{2}$,

$$
\begin{aligned}
\varphi^{c}\left(M_{1}\right. & \left.\# M_{2} ; V, W\right) \\
= & \left\langle e(V) Q_{2}^{\prime}(V) Q_{3}(W) Q_{1}\left(T M_{1} \# M_{2}\right) \hat{A}\left(M_{1} \# M_{2}\right),\left[M_{1} \# M_{2}\right]\right\rangle \\
= & \left\langle e\left(\bigoplus_{i=k_{2}+1}^{k_{1}} L_{j 1}\right)\left(e\left(\bigoplus_{i=1}^{k_{2}} L_{j 1}\right)+e\left(\bigoplus_{i=1}^{k_{2}} L_{j 2}\right)\right)\right. \\
& \left.\quad \times Q_{2}^{\prime}(V) Q_{3}(W) Q_{1}\left(T M_{1} \# M_{2}\right) \hat{A}\left(M_{1} \# M_{2}\right),\left[M_{1} \# M_{2}\right]\right\rangle .
\end{aligned}
$$

It follows from (3-2) that for $i>0$ the $i$-th Pontrjagin class of $W$ is given by $p_{i}\left(W_{1}\right)+p_{i}\left(W_{2}\right)$. A similar statement holds for the Chern classes of $V$.

Since $2^{-\operatorname{dim}_{\mathbb{C}} W} Q_{2}^{\prime}(V) Q_{3}(W) Q_{1}(T M) \hat{A}(M)$ is a power series with constant term one in the Pontrjagin classes of $V, W$ and $T M$ whose coefficients do not depend on $V, W$ and $T M$, it follows that, for $i>0$,

$$
\begin{aligned}
\left.2^{-\operatorname{dim}_{\mathbb{C} W}\left(Q_{2}^{\prime}(V)\right.} Q_{3}(W) Q_{1}\left(T M_{1} \# M_{2}\right) \widehat{A}\left(M_{1} \# M_{2}\right)\right)_{i} \\
=2^{-\operatorname{dim}_{\mathbb{C}} W_{1}}\left(Q_{2}^{\prime}\left(V_{1}\right) Q_{3}\left(W_{1}\right) Q_{1}\left(T M_{1}\right) \hat{A}\left(M_{1}\right)\right)_{i} \\
\quad+2^{-\operatorname{dim}_{\mathbb{C}} W_{2}}\left(Q_{2}^{\prime}\left(V_{2}\right) Q_{3}\left(W_{2}\right) Q_{1}\left(T M_{2}\right) \hat{A}\left(M_{2}\right)\right)_{i} .
\end{aligned}
$$

Here $\left(Q_{2}^{\prime}(V) Q_{3}(W) Q_{1}(T M)\right)_{i}$ denotes the degree $4 i$ part of $Q_{2}^{\prime}(V) Q_{3}(W) Q_{1}(T M)$. 
Now the statement follows from (3-2).

\section{Two vanishing results for the Witten genus}

In this section we prove vanishing results for the Witten genus of a Spin-manifold $M$ with $p_{1}(M)=0$ such that a product $M \times M^{\prime}$ admits an action of a compact connected semisimple Lie group of high rank.

Our first result is as follows.

Theorem 4.1 Let $M$ be a Spin-manifold such that $p_{1}(M)$ is torsion. Moreover, let $M^{\prime}$ be a $2 n$-dimensional $\mathrm{Spin}^{c}$-manifold such that there are $x_{1}, \ldots, x_{n} \in H^{2}\left(M^{\prime} ; \mathbb{Z}\right)$ with

(1) $\sum_{i=1}^{n} x_{i}=c_{1}^{c}\left(M^{\prime}\right)$ modulo torsion,

(2) $\sum_{i=1}^{n} x_{i}^{2}=p_{1}\left(M^{\prime}\right)$ modulo torsion,

(3) $\left\langle\prod_{i=1}^{n} x_{i},\left[M^{\prime}\right]\right\rangle \neq 0$.

If there is an almost effective action of a semisimple simply connected compact Lie group $G$ on $M \times M^{\prime}$ such that $\operatorname{rank} G>\operatorname{rank}\left\langle x_{1}, \ldots, x_{n}\right\rangle$, then the Witten genus $\varphi^{c}(M ; 0,0)$ of $M$ vanishes.

Proof Let $L_{i}, i=1, \ldots, n$, be the line bundle over $M^{\prime}$ with $c_{1}\left(L_{i}\right)=x_{i}$. By Lemma 2.7, the natural map $\iota^{*}: H_{G}^{2}\left(M \times M^{\prime} ; \mathbb{Z}\right) \rightarrow H^{2}\left(M \times M^{\prime} ; \mathbb{Z}\right)$ is an isomorphism.

Therefore by [10, Corollary 1.2] the $G$-action on $M \times M^{\prime}$ lifts into $p^{\prime *}\left(L_{i}\right), i=$ $1, \ldots, n$. Here $p^{\prime}: M \times M^{\prime} \rightarrow M^{\prime}$ is the projection. Moreover, by the above cited corollary and Lemma 2.1, the action of every $S^{1} \subset G$ lifts into the $\operatorname{Spin}^{c}$-structure on $M \times M^{\prime}$ induced by the Spin-structure on $M$ and the $\operatorname{Spin}^{c}$-structure on $M^{\prime}$.

By Lemma 3.1, we have

$$
\varphi^{c}\left(M \times M^{\prime} ; \bigoplus_{i=1}^{n} p^{\prime *} L_{i}, 0\right)=\varphi^{c}(M ; 0,0) \varphi^{c}\left(M^{\prime} ; \bigoplus_{i=1}^{n} L_{i}, 0\right) .
$$

By condition (3), we have

$$
\begin{aligned}
\varphi^{c}\left(M^{\prime} ; \bigoplus_{i=1}^{n} L_{i}, 0\right) & =\left\langle Q_{1}\left(T M^{\prime}\right) \prod_{i=1}^{n} x_{i} Q_{2}^{\prime}\left(\bigoplus_{i=1}^{n} L_{i}\right) \hat{A}\left(M^{\prime}\right),\left[M^{\prime}\right]\right\rangle \\
& =\left\langle\prod_{i=1}^{n} x_{i},\left[M^{\prime}\right]\right\rangle \neq 0 .
\end{aligned}
$$


Hence, $\varphi^{c}(M ; 0,0)$ vanishes if and only if $\varphi^{c}\left(M \times M^{\prime} ; \bigoplus_{i=1}^{n} p^{\prime *} L_{i}, 0\right)$ vanishes.

Let $T$ be a maximal torus of $G$. If there are no $T$-fixed points in $M \times M^{\prime}$, then the Lefschetz fixed point formula implies that this index vanishes. Therefore we may assume that there is a $T$-fixed point $y \in\left(M \times M^{\prime}\right)^{T}$.

As in the proof of Lemma 2.8 one proves that

$$
H^{4}(B G ; \mathbb{Q}) \longrightarrow H_{G}^{4}\left(M \times M^{\prime} ; \mathbb{Q}\right) \longrightarrow H^{4}\left(M \times M^{\prime} ; \mathbb{Q}\right)
$$

is exact. Therefore there is an $v \in H^{4}(B G ; \mathbb{Q})$ such that

$$
p_{1}^{T}\left(\bigoplus_{i=1}^{n} p^{\prime *} L_{i}-T\left(M \times M^{\prime}\right)\right)=\pi_{T}^{*} \rho(T, G)^{*} v .
$$

By Theorem 2.3, it is sufficient to show that there is a homomorphism $S^{1} \hookrightarrow T$ such that $\rho\left(S^{1}, T\right)^{*} \rho(T, G)^{*} v=a x^{2}$, where $x \in H^{2}\left(B S^{1} ; \mathbb{Z}\right)$ is a generator and $a \in \mathbb{Z}$, $a<0$.

We have

$$
\rho(T, G)^{*} v=\left.p_{1}^{T}\left(\bigoplus_{i=1}^{n} p^{*} L_{i}-T\left(M \times M^{\prime}\right)\right)\right|_{y}=\sum_{i=1}^{n} a_{i}^{2}-\sum v_{i}^{2},
$$

where $a_{i} \in H^{2}(B T ; \mathbb{Z}), i=1, \ldots, n$, are the weights of the $T$-representations $\left.p^{\prime *} L_{i}\right|_{y}$ and $v_{i} \in H^{2}(B T ; \mathbb{Z})$ are the weights of the $T$-representation $T_{y}\left(M \times M^{\prime}\right)$.

Since $\operatorname{rank} T>\operatorname{rank}\left\langle x_{1}, \ldots, x_{n}\right\rangle$ and

$$
a_{i}=\left.\left(\rho(T, G)^{*}\left(\iota^{*}\right)^{-1} p^{\prime *}\left(x_{i}\right)\right)\right|_{y} \quad \text { for } i=1, \ldots, n,
$$

there is a homomorphism $S^{1} \hookrightarrow T$ such that $\rho\left(S^{1}, T\right)^{*} a_{i}=0$ for $i=1, \ldots, n$.

For this $S^{1}$ we have $\rho\left(S^{1}, T\right)^{*} v=a x^{2}$ with $a \in \mathbb{Z}, a<0$, because the $G$-action is almost effective.

We will see later in Lemma 5.3 that those $2 n$-dimensional quasitoric manifolds whose orbit polytopes admit facet colorings with $n$ colors are examples of manifolds which satisfy the assumptions on $M^{\prime}$ in the above theorem. Other examples of such manifolds are given by those manifolds whose tangent bundle is isomorphic to a sum of complex line bundles and which have nonzero Euler characteristic. In particular, homogeneous spaces of the form $H / T$ with $H$ a semisimple compact connected Lie group and $T$ a maximal torus of $H$ are examples of such manifolds. Since in this case we have $b_{2}(H / T)=\operatorname{rank} H$ we get the following corollary. 
Corollary 4.2 Let $M$ be a Spin-manifold with $p_{1}(M)=0$ and $H$ a semisimple compact connected Lie group. If there is an almost effective action of a semisimple compact connected Lie group $G$ on $M \times H / T$ such that $\operatorname{rank} G>\operatorname{rank} H$, then the Witten genus of $M$ vanishes.

As an application of Corollary 4.2 we give a new proof for a theorem of Hauschild.

Corollary 4.3 [11, Theorem 9] Let $H$ be a semisimple compact connected Lie group with maximal torus $T$. Then we have $N^{s s}(H / T)=\operatorname{dim} H$.

Proof Let $G$ be a semisimple compact connected Lie group which acts effectively on $H / T$. Since the tangent bundle of $H / T$ splits as a sum of complex line bundles and $\chi(H / T) \neq 0$, there is a twisted Dirac operator with nonvanishing index on $H / T$. Therefore, by the first case in Corollary 2.13, we have

$$
\operatorname{dim} G-\operatorname{rank} G \leq \operatorname{dim} H / T=\operatorname{dim} H-\operatorname{rank} H .
$$

By Corollary 4.2 applied in the case $M=p t$, we see that $\operatorname{rank} G \leq \operatorname{rank} H$. Therefore it follows that $\operatorname{dim} G \leq \operatorname{dim} H$. Since there is an obvious action of $H$ on $H / T$, the statement follows.

Similarly to Theorem 4.1 we can prove the following vanishing result for actions of simple compact connected Lie groups of high rank.

Theorem 4.4 Let $M$ be a Spin-manifold such that $p_{1}(M)$ is torsion. Moreover, let $M^{\prime}$ be a $2 n$-dimensional $\mathrm{Spin}^{c}$-manifold such that $p_{1}\left(M^{\prime}\right)$ is torsion and there are $x_{1}, \ldots, x_{n} \in H^{2}\left(M^{\prime} ; \mathbb{Z}\right)$ and $1=n_{1}<n_{2}<\cdots<n_{k+1}=n+1$ with

(1) $\sum_{i=1}^{n} x_{i}=c_{1}^{c}\left(M^{\prime}\right)$ modulo torsion,

(2) $\sum_{i=n_{j}}^{n_{j+1}-1} x_{i}^{2}$ is torsion, for $j=1, \ldots, k$,

(3) $\left\langle\prod_{i=1}^{n} x_{i},\left[M^{\prime}\right]\right\rangle \neq 0$.

If there is an almost effective action of a simple simply connected compact Lie group $G$ on $M \times M^{\prime}$ such that $\operatorname{rank} G>\operatorname{rank}\left\langle x_{n_{j}}, \ldots, x_{n_{j+1}-1}\right\rangle$ for all $j=1, \ldots, k$, then the Witten genus $\varphi^{c}(M ; 0,0)$ of $M$ vanishes.

Proof The relations between the $G$-equivariant and nonequivariant cohomology of $M \times M^{\prime}$ is as described in the proof of Theorem 4.1. We consider the same index $\varphi^{c}\left(M \times M^{\prime} ; \bigoplus_{i=1}^{n} p^{\prime *} L_{i}, 0\right)$ as in the proof of that theorem. It vanishes if and only if the Witten genus of $M$ vanishes. 
Let $T$ be a maximal torus of $G$. We may assume that there is a $T$-fixed point $y$ in $M \times M^{\prime}$.

As in the proof of Theorem 4.1 one sees that there is an $v \in H^{4}(B G ; \mathbb{Q})$ such that $p_{1}^{T}\left(\bigoplus_{i=1}^{n} p^{\prime *} L_{i}-T\left(M \times M^{\prime}\right)\right)=\pi_{T}^{*} \rho(T, G)^{*} v$. By Theorem 2.3, it is sufficient to show that there is a homomorphism $S^{1} \hookrightarrow T$ such that $\rho\left(S^{1}, T\right)^{*} \rho(T, G)^{*} v=a x^{2}$, where $x \in H^{2}\left(B S^{1} ; \mathbb{Z}\right)$ is a generator and $a \in \mathbb{Z}, a<0$.

We have

$$
\rho(T, G)^{*} v=\left.p_{1}^{T}\left(\bigoplus_{i=1}^{n} p^{\prime *} L_{i}-T\left(M \times M^{\prime}\right)\right)\right|_{y}=\sum_{i=1}^{n} a_{i}^{2}-\sum v_{i}^{2},
$$

where $a_{i} \in H^{2}(B T ; \mathbb{Z}), i=1, \ldots, n$, are the weights of the $T$-representations $\left.p^{\prime *} L_{i}\right|_{y}$ and $v_{i} \in H^{2}(B T ; \mathbb{Z})$ are the weights of the $T$-representation $T_{y}\left(M \times M^{\prime}\right)$.

We will show that the $a_{i}, i=1, \ldots, n$, vanish.

Let $1 \leq j \leq k$. Since $H^{4}(B G ; \mathbb{Q}) \rightarrow H_{G}^{4}\left(M \times M^{\prime} ; \mathbb{Q}\right) \rightarrow H^{4}\left(M \times M^{\prime} ; \mathbb{Q}\right)$ is exact, there is an $v_{j}^{\prime} \in H^{4}(B G ; \mathbb{Q})$ such that

$$
p_{1}^{T}\left(\bigoplus_{i=n_{j}}^{n_{j}+1-1} p^{\prime *} L_{i}\right)=\pi_{T}^{*} \rho(T, G)^{*} v_{j}^{\prime}
$$

Therefore we have

$$
\sum_{i=n_{j}}^{n_{j}+1-1} a_{i}^{2}=\left.p_{1}^{T}\left(\bigoplus_{i=n_{j}}^{n_{j}+1-1} p^{\prime *} L_{i}\right)\right|_{y}=\rho(T, G)^{*} v_{j}^{\prime}
$$

Therefore $\sum_{i=n_{j}}^{n_{j+1}-1} a_{i}^{2}$ is invariant under the action of the Weyl group $W(G)$ of $G$ on $H^{4}(B T ; \mathbb{Q})$.

Because $\operatorname{dim} T>\operatorname{rank}\left\langle x_{n_{j}}, \ldots, x_{n_{j+1}-1}\right\rangle$ and $a_{i}=\left.\left(\rho(T, G)^{*}\left(\iota^{*}\right)^{-1} p^{\prime *}\left(x_{i}\right)\right)\right|_{y}$, there is an $S^{1} \subset T$ such that $\rho\left(S^{1}, T\right)^{*} a_{i}=0$ for $n_{j} \leq i \leq n_{j+1}-1$. Since $\sum_{i=n_{j}}^{n_{j+1}-1} a_{i}^{2} \in$ $H^{4}(B T ; \mathbb{Q})$ is $W(G)$-invariant, it follows that, for all $w \in W(G)$,

$$
0=\rho\left(w S^{1} w^{-1}, T\right)^{*} \sum_{i=n_{j}}^{n_{j+1}-1} a_{i}^{2}=\sum_{i=n_{j}}^{n_{j}+1-1}\left(\rho\left(w S^{1} w^{-1}, T\right)^{*} a_{i}\right)^{2} .
$$

Since $H^{*}\left(B S^{1} ; \mathbb{Z}\right)=\mathbb{Z}[x]$, this implies that $\rho\left(w S^{1} w^{-1}, T\right)^{*} a_{i}=0$ for all $n_{j} \leq i \leq$ $n_{j+1}-1$. Because $G$ is simple, there are no nontrivial $W(G)$-invariant subtori in $T$. Therefore we have $T=\left\langle w S^{1} w^{-1} ; w \in W(G)\right\rangle$. Hence, all $a_{i} \in H^{2}(B T ; \mathbb{Z})$, $i=n_{j}, \ldots, n_{j+1}-1, j=1, \ldots, k$, vanish. 
Hence, $\rho\left(S^{1}, T\right)^{*} \rho(T, G)^{*} v=a x^{2}$ with $a<0$ for all nontrivial homomorphisms $S^{1} \hookrightarrow T$.

Examples of those manifolds which satisfy the assumptions on $M^{\prime}$ in the above theorem are manifolds with nonvanishing Euler characteristic whose tangent bundle is isomorphic to a direct sum of complex line bundles $L_{1}, \ldots, L_{n}$ such that there are $1=n_{1}<n_{2}<\cdots<n_{k+1}=n+1$ with $p_{1}\left(\bigoplus_{i=n_{j}}^{n_{j+1}-1} L_{i}\right)=0$ for all $j=1, \ldots, k$.

If $H$ is a simple compact connected Lie group with maximal torus $T$, then all Pontrjagin classes of $H / T$ are torsion. Therefore we get the following corollary.

Corollary 4.5 Let $M$ be a Spin-manifold with $p_{1}(M)=0$ and $H_{1}, \ldots, H_{k}$ be simple compact connected Lie groups with maximal tori $T_{1}, \ldots, T_{k}$. If there is an almost effective action of a simple compact connected Lie group $G$ on $M \times \prod_{i=1}^{k} H_{i} / T_{i}$ such that rank $G>\operatorname{rank} H_{i}$ for all $i=1, \ldots, k$, then the Witten genus of $M$ vanishes.

The Corollaries 4.2 and 4.5 can be used to find an upper bound for the semisimple symmetry degree of $M \times H / T$, where $M$ is a Spin-manifold with $p_{1}(M)=0$ and nonvanishing Witten genus and $H$ is a semisimple compact Lie group with maximal torus $T$. To give this upper bound we need the following constants. For $l \geq 1$ let

$$
\alpha_{l}=\max \left\{\frac{\operatorname{dim} G}{\operatorname{rank} G} \mid G \text { a simple compact Lie group with } \operatorname{rank} G \leq l\right\} .
$$

The values of the $\alpha_{l}$ are listed in Table 1 .

\begin{tabular}{|c|c|c|}
\hline$l$ & $\alpha_{l}$ & $G_{l}$ \\
\hline 1 & 3 & $\operatorname{Spin}(3)$ \\
2 & 7 & $G_{2}$ \\
3 & 7 & $\operatorname{Spin}(7), \operatorname{Sp}(3)$ \\
4 & 13 & $F_{4}$ \\
5 & 13 & none \\
6 & 13 & $E_{6}, \operatorname{Spin}(13), \operatorname{Sp}(6)$ \\
7 & 19 & $E_{7}$ \\
8 & 31 & $E_{8}$ \\
$9 \leq l \leq 14$ & 31 & none \\
$l \geq 15$ & $2 l+1$ & $\operatorname{Spin}(2 l+1), \operatorname{Sp}(l)$ \\
\hline
\end{tabular}

Table 1: The values of $\alpha_{l}$ and the simply connected compact simple Lie groups $G_{l}$ of rank $l$ with $\operatorname{dim} G_{l}=\alpha_{l} \cdot l$ 
Corollary 4.6 Let $M$ be a Spin-manifold with $p_{1}(M)=0$, such that the Witten genus of $M$ does not vanish and $H_{1}, \ldots, H_{k}$ simple compact connected Lie groups with maximal tori $T_{1}, \ldots, T_{k}$. Then we have

$$
\sum_{i=1}^{k} \operatorname{dim} H_{i} \leq N^{s s}\left(M \times \prod_{i=1}^{k} H_{i} / T_{i}\right) \leq \alpha_{l} \sum_{i=1}^{k} \operatorname{rank} H_{i},
$$

where $l=\max \left\{\operatorname{rank} H_{i} \mid i=1, \ldots, k\right\}$. If all $H_{i}$ have the same rank and each $H_{i}$ has one of the groups listed in Table 1 as a covering group, then equality holds in both inequalities.

Proof Let $G$ be a compact simply connected semisimple Lie group which acts almost effectively on $M \times \prod_{i=1}^{k} H_{i} / T_{i}$. Then, by Corollary 4.2, we have $\operatorname{rank} G \leq$ $\sum_{i=1}^{k}$ rank $H_{i}$. By Corollary 4.5, all simple factors of $G$ must have rank smaller or equal to $l$. Therefore we have

$$
\operatorname{dim} G \leq \alpha_{l} \operatorname{rank} G \leq \alpha_{l} \sum_{i=1}^{k} \operatorname{rank} H_{i} .
$$

Hence, $N^{s s}\left(M \times \prod_{i=1}^{k} H_{i} / T_{i}\right) \leq \alpha_{l} \sum_{i=1}^{k}$ rank $H_{i}$ follows.

Since there is an obvious $\prod_{i=1}^{k} H_{i}$-action on $M \times \prod_{i=1}^{k} H_{i} / T_{i}$, the other inequality follows.

If all $H_{i}$ have the same rank $l$ and each $H_{i}$ has one of the groups listed in Table 1 as a covering group, then $\sum_{i=1}^{k} \operatorname{dim} H_{i}=\alpha_{l} \sum_{i=1}^{k}$ rank $H_{i}$. Therefore we get equality in this case.

Remark 4.7 Our methods to prove Corollary 4.6 break down if we consider the stabilization of $M$ with a homogeneous space $H / K$, where $K$ is a closed subgroup of $H$ which is not a maximal torus.

If $K$ has not maximal rank in $H$, then there is a fixed-point-free torus action on $M \times H / K$. Therefore all indices $\varphi^{c}(M \times H / K ; V, W)$ vanish by the Lefschetz fixed point formula. If $K$ is nonabelian and has maximal rank in $H$, then all indices $\varphi^{c}(M \times H / K ; V, W)$ vanish by Corollary 2.11 .

Therefore in both cases the starting point of the proofs of Theorems 4.1 and 4.4, namely the existence of an index $\varphi^{c}\left(M \times M^{\prime} ; V, W\right)$ which vanishes if and only if the Witten genus of $M$ vanishes, does not hold in the case $M^{\prime}=H / K$. 


\section{Twisted Dirac operators and quasitoric manifolds}

In this section we apply the results of the previous sections to the study of quasitoric manifolds. We begin by recalling the definition of quasitoric manifolds and some of their properties established by Davis and Januszkiewicz [6] (see also Buchstaber and Panov [5]).

A smooth closed simply connected $2 n$-dimensional manifold $M$ with a smooth action of an $n$-dimensional torus $T$ is called quasitoric if the following two conditions are satisfied:

(1) The $T$-action on $M$ is locally isomorphic to the standard action of $T$ on $\mathbb{C}^{n}$.

(2) The orbit space $M / T$ is a simple convex $n$-dimensional polytope $P$.

We denote by $\mathfrak{F}=\left\{F_{1}, \ldots, F_{m}\right\}$ the set of facets of $P$. Then for each $F_{i} \in \mathfrak{F}$, $M_{i}=\pi^{-1}\left(F_{i}\right)$ is a closed connected submanifold of codimension two in $M$ which is fixed pointwise by a one-dimensional subtorus $\lambda\left(F_{i}\right)=\lambda\left(M_{i}\right)$ of $T$. Here $\pi: M \rightarrow P$ denotes the orbit map. These $M_{i}$ are called the characteristic submanifolds of $M$.

The cohomology ring of $M$ is generated by elements of degree two $u_{1}, \ldots, u_{m} \in$ $H^{2}(M ; \mathbb{Z})$ such that

$$
H^{*}(M ; \mathbb{Z})=\mathbb{Z}\left[u_{1}, \ldots, u_{m}\right] /(I+J),
$$

where $I$ is the ideal generated by

$$
\left\{\prod_{j=1}^{k} u_{i_{j}} ; \bigcap_{j=1}^{k} F_{i_{j}}=\varnothing\right\}
$$

and $J$ is generated by linear relations between the $u_{i}$, which depend on the function $\lambda: \mathfrak{F} \rightarrow\{$ one-dimensional subtori of $T\}$. It should be noted that each $u_{i}$ is the Poincaré dual of $M_{i}$.

The stable tangent bundle of $M$ splits as a sum of complex line bundles $L_{1}, \ldots, L_{m}$ :

$$
T M \oplus \mathbb{R}^{2 m-2 n} \cong \bigoplus_{i=1}^{m} L_{i},
$$

such that $c_{1}\left(L_{i}\right)= \pm u_{i}$. In particular, a quasitoric manifold has always a stable almost complex structure and therefore a $\operatorname{Spin}^{c}$-structure.

So the results of Section 2 might be used to find quasitoric manifolds with only a few nonabelian symmetries. To do so, we have to find quasitoric manifolds $M$ that admit 
vector bundles $V \rightarrow M$ and $W \rightarrow M$ which satisfy the assumptions of Theorem 2.4 such that $\varphi^{c}(M ; V, W) \neq 0$. In the following we say that $M$ has a nonvanishing index $\varphi^{c}(M ; V, W)$ if these assumptions are satisfied.

Now we turn to the construction of such quasitoric manifolds. Because the stable tangent bundle of a quasitoric manifold $M$ splits as a sum of line bundles $\bigoplus_{i=1}^{m} L_{i}$ it seems to be natural to consider indices $\varphi^{c}(M ; V, W)$ with $V=\bigoplus_{i=1}^{k} L_{i}, W=\bigoplus_{i=k+1}^{m} L_{i}$ and $W$ a Spin-bundle. But we have the following result:

Theorem 5.1 Let $M$ be quasitoric. Moreover, let $M_{1}, \ldots, M_{m}$ be the characteristic submanifolds of $M$ and $L_{i} \rightarrow M$ the complex line bundles with $c_{1}\left(L_{i}\right)=P D\left(M_{i}\right)$. Let

$$
V=\bigoplus_{i=1}^{k} L_{i} \quad \text { and } \quad W=\bigoplus_{i=k+1}^{m} L_{i}
$$

with $c_{1}(V) \equiv c_{1}(M) \bmod 2$ and $c_{1}(W) \equiv 0 \bmod 2$. Let $\partial_{c}$ be the Dirac operator for a Spin ${ }^{c}$-structure on $M$ with $c_{1}^{c}(M)=c_{1}(V)$. Then $\varphi^{c}(M ; V, W)=0$.

Proof We have

$$
\begin{aligned}
\varphi^{c}(M ; V, W) & =\left\langle e^{c_{1}^{c}(M) / 2} \operatorname{ch}(R) \hat{A}(M),[M]\right\rangle \\
& =\left\langle e(V) Q_{1}(T M) Q_{2}^{\prime}(V) Q_{3}(W) \widehat{A}(V \oplus W),[M]\right\rangle \\
& =\left\langle Q_{1}(V) Q_{1}(W) Q_{2}^{\prime}(V) Q_{3}(W) \hat{A}(V) \widehat{A}(W),[N]\right\rangle \\
& =\left\langle Q_{1}(W) Q_{3}(W) \widehat{A}(W),[N]\right\rangle \\
& =2^{m-n} \varphi^{c}(N ; 0, T N)=0 .
\end{aligned}
$$

Here $N$ is the intersection $\bigcap_{i=1}^{k} M_{i}$, which is a quasitoric Spin-manifold. $N$ can not be a point, since otherwise the first Chern classes of the summands of $W$ form a basis of $H^{2}(M ; \mathbb{Z})$. Therefore $W$ cannot be Spin if $N$ is a point. The elliptic genus $\varphi^{c}(N ; 0, T N)$ of $N$ vanishes, because there is an odd $S^{1}$-action on $N$ (see Hirzebruch and Slodowy [12, page 317]).

So we need another idea to construct quasitoric manifolds $M$ which have nontrivial indices $\varphi^{c}(M ; V, W)$. We will prove that those $2 n$-dimensional quasitoric manifolds whose orbit polytopes admit facet colorings with $n$ colors are such examples. Before we do so we summarize some properties of $n$-dimensional polytopes and facet colorings.

A facet coloring with $d$ colors of a simple $n$-dimensional polytope $P$ is a map $f: \mathfrak{F} \rightarrow\{1, \ldots, d\}$ such that $f\left(F_{i}\right) \neq f\left(F_{j}\right)$ whenever $F_{i} \cap F_{j} \neq \varnothing$ and $F_{i} \neq F_{j}$. Because in each vertex of $P$ there meet $n$ facets, one needs at least $n$ colors to color $P$. 
The following description of simple $n$-dimensional polytopes which admit a coloring with $n$ colors is due to Joswig.

Theorem 5.2 [13, Theorem 16] Let $P$ be a simple $n$-dimensional polytope. Then the following statements are equivalent:

(1) $P$ is even, ie each two-dimensional face of $P$ has an even number of vertices.

(2) The graph which consists out of the vertices and edges of $P$ is bipartite.

(3) The boundary complex $\partial P^{*}$ of the dual polytope of $P$ is balanced, ie there is a nondegenerate simplicial map $\partial P^{*} \rightarrow \Delta^{n-1}$. Here $\Delta^{n-1}$ denotes the $(n-1)-$ dimensional simplex.

(4) $P$ admits a facet coloring with $n$ colors.

Quasitoric manifolds whose orbit polytopes satisfy condition (3) in the above theorem were described by Davis and Januszkiewicz [6, pages 425-426]. They show that this is a very rich class of quasitoric manifolds. We should note that the $n$-dimensional cube admits a facet coloring with $n$ colors. Moreover, a simple polytope belongs to this class if $\partial P^{*}$ is the barycentric subdivision of a convex polytope.

Now we construct a nonvanishing index $\varphi^{c}(M ; V, W)$ on every $2 n$-dimensional quasitoric manifold $M$ whose orbit polytope admits a facet coloring with $n$ colors.

Lemma 5.3 Let $M$ be a quasitoric manifold of dimension $2 n$ over the polytope $P$. Assume that $P$ admits a facet coloring with $n$ colors. Then there is a $\operatorname{Spin}^{c}$-structure on $M$ and a complex vector bundle $V$ which is a sum of line bundles with $c_{1}(V)=c_{1}^{c}(M)$ and $p_{1}(M)=p_{1}(V)$, such that $\varphi^{c}(M ; V, 0)$ does not vanish.

Proof Let $f: \mathfrak{F} \rightarrow\{1, \ldots, n\}$ be a facet coloring of $P$ with $n$ colors.

Let $V=\bigoplus_{i=1}^{n} L_{f^{-1}(i)}$, where $L_{f^{-1}(i)}$ is the line bundle with

$$
c_{1}\left(L_{f^{-1}(i)}\right)=\sum_{F_{j} \in f^{-1}(i)} \pm u_{j} .
$$

Then we have $c_{1}(V) \equiv c_{1}(M) \bmod 2$ and

$$
p_{1}(V)=\sum_{i=1}^{n}\left(\sum_{F_{j} \in f^{-1}(i)} \pm u_{j}\right)^{2}=\sum_{i=1}^{n} \sum_{F_{j} \in f^{-1}(i)} u_{j}^{2}=p_{1}(M) .
$$


Consider a Spin ${ }^{c}$-structure on $M$ with $c_{1}^{c}(M)=c_{1}(V)$ and assume that the associated index $\varphi^{c}(M ; V, 0)$ vanishes. Then $\varphi^{c}(M ; V, 0)$ may be calculated as:

$$
\begin{aligned}
0 & =\left\langle Q_{1}(T M) \prod_{i=1}^{n} c_{1}\left(L_{f^{-1}(i)}\right) Q_{2}^{\prime}(V) \widehat{A}(M),[M]\right\rangle \\
& =\left\langle\prod_{i=1}^{n} c_{1}\left(L_{f^{-1}(i)}\right),[M]\right\rangle .
\end{aligned}
$$

Therefore we have

$$
\prod_{i=1}^{n} \sum_{F_{j} \in f^{-1}(i)} \pm u_{j}=0
$$

Since the signs of the $u_{j}$ may be changed freely, we get, by considering different $\operatorname{Spin}^{c}$-structures and summing up in Equation (5-1):

$$
\forall\left(F_{i_{1}}, \ldots, F_{i_{n}}\right) \in f^{-1}(1) \times \cdots \times f^{-1}(n), \quad \prod_{j=1}^{n} u_{i_{j}}=0 .
$$

But there is at least one tuple $\left(F_{i_{1}}, \ldots, F_{i_{n}}\right) \in \mathfrak{F} \times \cdots \times \mathfrak{F}$ such that $\bigcap_{j=1}^{n} F_{i_{j}}$ is a vertex of $P$. For this tuple we have $\prod_{j=1}^{n} u_{i_{j}} \neq 0$. Moreover, because $f$ is a coloring with $n$ colors, for each $k \in\{1, \ldots, n\}$ there is exactly one $F_{i_{j_{k}}}$ with $f\left(F_{i_{j_{k}}}\right)=k$. Therefore we get a contradiction.

As a consequence of Lemma 5.3 and the corollaries at the end of Section 2 we get the following corollaries.

Corollary 5.4 Let $M$ be a $2 n$-dimensional quasitoric manifold. If the orbit polytope of $M$ admits a facet coloring with $n$ colors, then we have $N(M) \leq 3 n$ with equality holding if and only if $M=\prod S^{2}$.

Proof This follows directly from Lemma 5.3 and Corollary 2.14.

Corollary 5.5 Let $M$ be a quasitoric manifold over the $n$-dimensional cube. Then the only simple simply connected compact Lie groups that can act almost effectively on $M$ are $\mathrm{SU}(2)$ and $\operatorname{Spin}(5)$.

Proof By Lemma 5.3, there is a twisted Dirac operator on $M$, whose index does not vanish. By Corollary 2.12, the order of the Weyl group of a simple simply connected compact Lie group, which acts on $M$, divides the Euler characteristic of $M$. Because $\chi(M)=2^{n}$ and $\mathrm{SU}(2)$ and $\mathrm{Spin}(5)$ are the only simple simply connected compact Lie

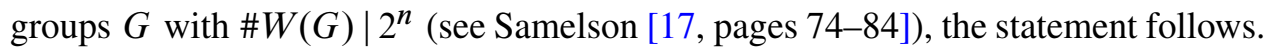


In the proof of the next corollary of Lemma 5.3 we construct quasitoric manifolds with low semisimple symmetry degree.

Corollary 5.6 In each dimension greater or equal to four, there are infinitely many quasitoric manifolds $M$ with $N^{s s}(M) \leq 3$.

Proof Let $M_{1}$ be a four-dimensional quasitoric manifold over a polygon with $6 \mathrm{k}$ vertices, $k \in \mathbb{N}$. Moreover, let $M_{2}$ be a $2 n$-dimensional quasitoric manifold over the $n$-cube. Then the orbit polytopes of $M_{1}$ and $M_{2}$ admit facet colorings with 2 and $n$ colors, respectively. Therefore the orbit polytope of $M_{1} \times M_{2}$ admits a facet coloring with $n+2$ colors. Hence, by Lemma 5.3, there is a nonvanishing index $\varphi^{c}\left(M_{1} \times M_{2} ; V, 0\right)$ on $M_{1} \times M_{2}$. By Lemma 3.2 applied in the case $V_{1}=V_{2}=V$ and $W_{1}=W_{2}=0$, it follows that

$$
M=\left(M_{1} \times M_{2}\right) \#\left(M_{1} \times M_{2}\right)
$$

has a nonvanishing index. Because $\chi(M)=2 \cdot 6 k \cdot 2^{n}-2$ is not divisible by three and four, it follows from Corollary 2.12 and [17, pages 74-84] that the only compact simply connected semisimple Lie group that can act almost effectively on $M$ is $\mathrm{SU}(2)$. Because connected sums of quasitoric manifolds are quasitoric, the statement follows.

The connected sum of two quasitoric manifolds is again a quasitoric manifold. Therefore Lemma 5.3 and the following result may be used to construct more quasitoric manifolds with nonvanishing indices.

Lemma 5.7 Let $M_{1}, M_{2}$ be quasitoric manifolds of dimension $2 n \geq 4$. Assume that there are vector bundles $V_{1} \rightarrow M_{1}$ and $W_{1} \rightarrow M_{1}$ as in Lemma 3.2 and $b_{2}\left(M_{2}\right) \leq$ $\operatorname{dim} V_{1}$ or $M_{2}$ is a Spin-manifold.

Then there are sums of line bundles $V, W$ over $M_{1} \# M_{2}$ and a $\operatorname{Spin}^{c}$-structure on $M_{1} \# M_{2}$ with $c_{1}(V)=c_{1}^{c}\left(M_{1} \# M_{2}\right), c_{1}(W) \equiv 0 \bmod 2, p_{1}\left(V+W-T M_{1} \# M_{2}\right)=0$, such that

$$
\varphi^{c}\left(M_{1} \# M_{2} ; V, W\right)=2^{k} \varphi^{c}\left(M_{1} ; V_{1}, W_{1}\right)
$$

for some $k \geq 0$.

Proof Let $L_{i} \rightarrow M_{2}, i=1, \ldots, m$, be line bundles such that the Chern classes of the $L_{i}$ are the Poincaré duals of the characteristic submanifolds of $M_{2}$. Then we have $T M_{2} \oplus \mathbb{R}^{2 m-2 n} \cong \bigoplus_{i=1}^{m} L_{i}$. 
We order the $L_{i}$ so that $c_{1}\left(L_{1}\right), \ldots, c_{1}\left(L_{b_{2}\left(M_{2}\right)}\right)$ form a basis of $H^{2}\left(M_{2} ; \mathbb{Z}\right)$. Therefore there are $a_{1}, \ldots, a_{b_{2}(M)} \in\{0,1\}$ such that

$$
c_{1}\left(\bigoplus_{i=1}^{b_{2}(M)} a_{i} L_{i}\right) \equiv c_{1}\left(M_{2}\right) \bmod 2
$$

and

$$
W_{2}=\bigoplus_{i=1}^{b_{2}(M)}\left(1-a_{i}\right) L_{i} \oplus \bigoplus_{i=b_{2}(M)+1}^{m} L_{i}
$$

is a Spin bundle. Consider a $\operatorname{Spin}^{c}$-structure on $M_{2}$ such that

$$
c_{1}^{c}\left(M_{2}\right)=c_{1}\left(\bigoplus_{i=1}^{b_{2}(M)} a_{i} L_{i}\right) .
$$

By Theorem 5.1 we have $\varphi^{c}\left(M_{2} ; V_{2}, W_{2}\right)=0$, where $V_{2}=\bigoplus_{i=1}^{b_{2}(M)} a_{i} L_{i}$. Therefore, by Lemma 3.2, the statement follows.

In dimensions divisible by four we can use Lemma 5.7 to improve the results of Corollary 5.6 and prove that there are quasitoric manifolds on which no semisimple compact Lie group can act effectively.

Corollary 5.8 In dimensions $4 k, k>0$, there are infinitely many quasitoric manifolds $M$ with $N^{s s}(M)=0$.

Proof Let $M^{\prime}$ be as in Lemma 5.3 with $\operatorname{dim} M^{\prime}=2 n=4 k$. Then, by an iterated application of Lemma 5.7, there are nonvanishing indices $\varphi^{c}(M ; V, W)$ on the manifold $M=M^{\prime} \# l \mathbb{C P}^{2 k}$ with $l \in \mathbb{N}$. Because connected sums of quasitoric manifolds are quasitoric, $M$ is quasitoric.

Since a bipartite regular graph has an even number of vertices, Theorem 5.2 gives that the Euler characteristic of $M^{\prime}$ is even. Therefore $\chi(M)=\chi\left(M^{\prime}\right)+l \chi\left(\mathbb{C P}^{n}\right)-2 l$ is odd if $l$ is odd. Because the order of the Weyl group of a semisimple compact connected Lie group is even [17, pages 74-84], the statement follows from Corollary 2.12.

Remark 5.9 Nonsingular projective toric varieties are examples of quasitoric manifolds. If, in the situation of the proof of Corollary $5.8, M^{\prime}$ is such a variety, then we can construct infinitely many nonsingular toric varieties $M$ with $N^{s S}(M)=0$ by blowing-up isolated fixed points in $M^{\prime}$ repeatedly, ie by taking connected sums with several copies of $\overline{\mathbb{C P}}^{n}$. 


\section{Quasitoric manifolds admitting low cohomogeneity actions}

In this section we study quasitoric manifolds which admit a cohomogeneity one or zero action of a compact connected Lie group and have a nonzero index $\varphi^{c}(M ; V, W)$. To do so we need the notion of spaces of $q$-type which was introduced by Hauschild [11]. A space of $q$-type is defined to be a topological space $X$ satisfying the following cohomological properties:

- The cohomology ring $H^{*}(X ; \mathbb{Q})$ is generated as a $\mathbb{Q}$-algebra by elements of degree two, ie $H^{*}(X ; \mathbb{Q})=\mathbb{Q}\left[x_{1}, \ldots, x_{n}\right] / I_{0}$ and $\operatorname{deg} x_{i}=2$.

- The defining ideal $I_{0}$ contains a definite quadratic form $Q$.

Examples of spaces of $q$-type are homogenous spaces of the form $G / T$ where $G$ is a semisimple compact connected Lie group and $T$ a maximal torus of $G$. Quasitoric manifolds of $q$-type were studied by the author in [19].

For the proof of the main result of this section we need the following lemma.

Lemma 6.1 Let $F \rightarrow E \rightarrow B$ be a fibration such that $\pi_{1}(B)$ acts trivially on $H^{*}(F ; \mathbb{Q})$. If $F$ and $B$ are spaces of $q$-type then $E$ is a space of $q$-type.

Proof Because $H^{*}(F ; \mathbb{Q})$ and $H^{*}(B ; \mathbb{Q})$ are generated by their degree two parts, it follows from the Serre spectral sequence that $H^{*}(E ; \mathbb{Q})$ is generated by its degree two part. Let $x_{1}, \ldots, x_{m}$ be a basis of $H^{2}(F ; \mathbb{Q})$ and $y_{1}, \ldots, y_{m^{\prime}}$ be a basis of $H^{2}(B ; \mathbb{Q})$. Then there is a basis $X_{1}, \ldots, X_{m}, Y_{1}, \ldots, Y_{m^{\prime}}$ of $H^{2}(E ; \mathbb{Q})$ such that $\iota^{*} X_{i}=x_{i}, i=1, \ldots, m$, and $\pi^{*} y_{i}=Y_{i}, i=1, \ldots, m^{\prime}$. Here $\iota: F \rightarrow E$ is the inclusion and $\pi: E \rightarrow B$ is the projection.

Let $Q_{F}$ and $Q_{B}$ be positive definite bilinear forms such that $Q_{F}\left(x_{1}, \ldots, x_{m}\right)=0 \in$ $H^{4}(F ; \mathbb{Q})$ and $Q_{B}\left(y_{1}, \ldots, y_{m^{\prime}}\right)=0 \in H^{4}(B ; \mathbb{Q})$.

Then there are $\alpha_{11}, \ldots, \alpha_{m m^{\prime}} \in \mathbb{Q}$ and $\beta_{1}, \ldots, \beta_{m^{\prime}} \in \mathbb{Q}$ such that for all $\lambda \in \mathbb{Q}$ :

$Q_{\lambda}\left(X_{1}, \ldots, X_{m}, Y_{1}, \ldots, Y_{m^{\prime}}\right)$

$$
\begin{aligned}
& =Q_{F}\left(X_{1}, \ldots, X_{m}\right)+\lambda Q_{B}\left(Y_{1}, \ldots, Y_{m^{\prime}}\right)+\sum_{i, j} \alpha_{i j} X_{i} Y_{j}+\sum_{i} \beta_{i} Y_{i}^{2} \\
& =0 \in H^{4}(E ; \mathbb{Q}) .
\end{aligned}
$$

We claim that $Q_{\lambda}$ is positive definite for sufficient large $\lambda$. To see that it is sufficient to show that for all $a \in S^{m+m^{\prime}-1} \subset \mathbb{R}^{m+m^{\prime}}, Q_{\lambda}(a)>0$. We may write $a=\gamma_{1} x+\gamma_{2} y$, with $x \in \mathbb{R}^{m}, y \in \mathbb{R}^{m^{\prime}},\|x\|=\|y\|=1$ and $\gamma_{1}^{2}+\gamma_{2}^{2}=1$. 
Because $Q_{F}$ is positive definite and $S^{m+m^{\prime}-1} \cap \mathbb{R}^{m}$ is compact, there is an $\epsilon>0$ such that $Q_{\lambda}(a)-\lambda Q_{B}(a)>0$ for all $\gamma_{2}<\epsilon$.

Because $Q_{B}$ is positive definite and $S^{m+m^{\prime}-1} \cap\left\{\gamma_{2} \geq \epsilon\right\}$ is compact, we may take $\lambda$ sufficiently large such that

$$
\begin{aligned}
\lambda \min \left\{Q_{B}(a) \mid a \in S^{m+m^{\prime}-1}\right. & \left.\cap\left\{\gamma_{2} \geq \epsilon\right\}\right\} \\
& >-\min \left\{Q_{\lambda}(a)-\lambda Q_{B}(a) \mid a \in S^{m+m^{\prime}-1} \cap\left\{\gamma_{2} \geq \epsilon\right\}\right\} .
\end{aligned}
$$

Therefore $Q_{\lambda}$ is positive definite for sufficient large $\lambda$.

Now we can prove the following theorem.

Theorem 6.2 Let $M$ be a quasitoric manifold on which a compact connected Lie group $G$ acts such that $\operatorname{dim} M / G \leq 1$. Assume that $M$ has a nonvanishing index $\varphi^{c}(M ; V, W)$ with $V, W$ as in Theorem 2.4.

Then $M=\prod S^{2}$ if $\operatorname{dim} M / G=0$ or $M$ is a $S^{2}$-bundle with structure group a maximal torus of $G$ over $\prod S^{2}$ if $\operatorname{dim} M / G=1$.

Proof If $\operatorname{dim} M / G=0$ then $M$ is a homogeneous space $G / H$. Because $\chi(M) \neq 0$, $H$ must have maximal rank in $G$. Therefore we may assume that $G$ is semisimple. Hence, it follows from Corollary 2.11 that $H$ is a maximal torus of $G$. As in [19, Section 3], one sees that $M=\prod S^{2}$.

Now assume that $\operatorname{dim} M / G=1$. Because $\chi(M) \neq 0$, it follows from Corollary 2.11 that there is an orbit of type $G / T$ with $T$ a maximal torus of $G$. Because $\operatorname{dim} G / T$ is even this must be a nonprincipal orbit. Hence, the orbit space $M / G$ is homeomorphic to the compact interval $[0,1]$ and there is exactly one other nonprincipal orbit. Let $S \subset G$ be a principal isotropy group. Then we may assume $S \subset T$. Moreover, $T / S$ is a sphere. Therefore $S$ has codimension one in $T$.

Let $K^{+}$be the isotropy group of the other nonprincipal orbit. Then $K^{+} / S$ is a sphere and the identity component of $K^{+}$is a torus by Theorem 2.4. Therefore there are two cases:

- $\operatorname{dim} K^{+}=\operatorname{dim} S$ and $K^{+} / S=\mathbb{Z}_{2}$.

- $K^{+}$is a maximal torus of $G$.

In the first case, we have by the Seifert-van Kampen theorem

$$
\pi_{1}(M)=\pi_{1}(G / T) *_{\pi_{1}(G / S)} \pi_{1}\left(G / K^{+}\right)=\pi_{1}\left(G / K^{+}\right) / \pi_{1}(G / S)=\mathbb{Z}_{2}
$$


because $G / S \rightarrow G / K^{+}$is a twofold covering. But $M$ is simply connected because it is quasitoric. So this case does not occur.

Now as in the remark before [19, Lemma 5.2] one sees that $M$ is a $S^{2}$-bundle with structure group $T$ over $G / T$. By Lemma $6.1, M$ is a quasitoric manifold which is of $q$-type. Therefore it follows from [19, Theorem 5.3] that $M$ is a $S^{2}$-bundle over $\prod S^{2}$.

\section{Quasitoric manifolds with nonvanishing indices and $N(M) \geq 3 n-4$}

By Corollary 2.14 the symmetry degree of a quasitoric manifold $M$ with a nontrivial index $\varphi^{c}(M ; V, W)$ is bounded from above by $3 n$. In this section we classify those $2 n$-dimensional quasitoric manifolds which admit a twisted Dirac operator with a nonvanishing index and have degree of symmetry greater or equal to $3 n-4$.

For the statement of our first theorem we need the notion of a torus manifold. A torus manifold is a $2 n$-dimensional closed connected orientable smooth manifold $M$ with an effective smooth action of an $n$-dimensional torus $T$, such that $M^{T}$ is nonempty.

Theorem 7.1 Let $M$ be a $2 n$-dimensional quasitoric manifold with nonvanishing index $\varphi^{c}(M ; V, W)$ with $V, W$ as in Theorem 2.4 and $G$ be a compact connected Lie group of rank $n$, which acts almost effectively on $M$. Then $G$ has a covering group of the form $\prod \mathrm{SU}(2) \times T^{l_{0}}$. Moreover, $M$ is a fiber bundle with fiber a $2 l_{0}$-dimensional torus manifold over $\prod S^{2}$.

Proof $M$ is a torus manifold with $G$-action in the sense of the author's [18] and $H^{*}(M ; \mathbb{Z})$ is generated by $H^{2}(M ; \mathbb{Z})$. Therefore $G$ has a covering group of the form $\widetilde{G}=\prod_{i} \operatorname{SU}\left(l_{i}+1\right) \times T^{l_{0}}$ by [18, Remark 2.9]. Let $T$ be a maximal torus of $G$ and $x \in M^{T}$. Then, by [18, Lemmas 3.1 and 3.4], we have

$$
\mathrm{SU}\left(l_{i}+1\right)_{x}=S\left(U\left(l_{i}\right) \times U(1)\right) \quad \text { or } \quad \mathrm{SU}\left(l_{i}+1\right)_{x}=\mathrm{SU}\left(l_{i}+1\right) .
$$

Therefore by Corollary 2.11 , we have $l_{i}=1$. Moreover, each factor $\mathrm{SU}\left(l_{i}+1\right)$ does not have a fixed point in $M$. Therefore the second statement follows from an iterated application of [18, Corollary 5.6].

The next theorem is the classification announced in the introduction of this section.

Theorem 7.2 Let $M$ be a $2 n$-dimensional quasitoric manifold with nonvanishing index $\varphi^{c}(M ; V, W)$ with $V, W$ as in Theorem 2.4. If $N(M) \geq 3 n-4$, then $M$ is diffeomorphic to one of the manifolds in the following list: 


\begin{tabular}{|c|c|}
\hline$N(M)$ & $M$ \\
\hline $3 n$ & $\prod S^{2}$ \\
$3 n-1$ & impossible \\
$3 n-2$ & $S^{2}$-bundle over $\prod S^{2}$ \\
$3 n-3$ & impossible \\
$3 n-4$ & $N$-bundle over $\prod S^{2}$ with $N$ a \\
& quasitoric manifold, $\operatorname{dim} N=4$ \\
\hline
\end{tabular}

Proof The statement about the quasitoric manifolds with $N(M)=3 n$ follows from Corollary 2.14 .

Therefore assume that $M$ is a $2 n$-dimensional quasitoric manifold with nonvanishing index $\varphi^{c}(M ; V, W)$ and $G$ is a compact connected Lie group of dimension $3 n-1$, which acts effectively on $M$. Let $T$ be a maximal torus of $G$.

Because, by Corollary 2.13,

$$
\operatorname{dim} G-\operatorname{dim} T \leq 2 n,
$$

we have $\operatorname{dim} T=n-1$ or $\operatorname{dim} T=n$. If $\operatorname{dim} T=n$, then $\operatorname{dim} G-\operatorname{dim} T$ is odd, which is impossible. But $\operatorname{dim} T=n-1$ is impossible by Corollary 2.13.

Let $M, G, T$ as above. But with $\operatorname{dim} G=3 n-2$. By (7-1), we have $\operatorname{dim} T=$ $n-2, n-1, n$. As in the first case one sees that $\operatorname{dim} T=n-2, n-1$ are impossible. If $\operatorname{dim} T=n$, we see with Theorem 7.1 that $M$ is a $S^{2}$-bundle over $\prod S^{2}$.

Let $M, G, T$ as above. But with $\operatorname{dim} G=3 n-3$. By (7-1), we have $\operatorname{dim} T=$ $n-3, n-2, n-1, n$. As above one sees that $\operatorname{dim} T=n-3, n-2, n$ are impossible. Therefore we have $\operatorname{dim} T=n-1$. Because $\chi(M) \neq 0$, there is by Corollary 2.11 an orbit of type $G / T$ which has dimension $2 n-2$.

Therefore the principal orbit type has dimension $2 n-2$ or $2 n-1$. In the first case the principal orbit type is $G / T$ and by Corollary 2.11 there is no exceptional or singular orbit. Hence, $M$ is a fiber bundle over a simply connected surface with fiber $G / T$ and structure group $N_{G} T / T$. Since $N_{G} T / T$ is finite, we have $M=S^{2} \times G / T$. Therefore we have $N(M) \geq 3+\operatorname{dim} G=3 n$.

Now assume that the principal orbit $G / S$ has codimension one in $M$. Then, by Theorem 6.2, $M$ is a $S^{2}$-bundle with structure group a torus over $\prod_{i=1}^{n-1} S^{2}$. Therefore we have $N(M) \geq 3 n-2$.

Now let $M, G, T$ as above, but with $\operatorname{dim} G=3 n-4$. By (7-1), we have $\operatorname{dim} T=$ $n-4, n-3, n-2, n-1, n$. As above one sees that $\operatorname{dim} T=n-4, n-3, n-1$ are impossible. Therefore we have $\operatorname{dim} T=n-2, n$. 
At first assume that $\operatorname{dim} T=n$. Then $M$ is a torus manifold with $G$-action. By Theorem 7.1 we have $G=\prod_{i=1}^{k} \mathrm{SU}(2) \times T^{l_{0}}$ with $3 n-4=3 k+l_{0}$ and $n=k+l_{0}$. Therefore we have $l_{0}=2$ and $M$ is a fiber bundle with fiber a four-dimensional torus manifold over $\prod_{i=1}^{k} S^{2}$. By [18, Lemma 5.17], the fiber of this bundle is simply connected. Therefore it is quasitoric because every four-dimensional simply connected torus manifold is quasitoric (see Orlik and Raymond [15, Section 5]).

Now assume that $\operatorname{dim} T=n-2$. Then we have $\operatorname{dim} G / T=2 n-2$. Therefore the principal orbit type of the $G$-action on $M$ has dimension $2 n-2$ or $2 n-1$. In both cases one sees as in the case $\operatorname{dim} G=3 n-3$ that $N(M) \geq 3 n-2$.

\section{Highly symmetric quasitoric manifolds}

In this section we show that $\mathbb{C P}^{n}$ is the most symmetric quasitoric manifold of dimension $2 n$. The main result of this section is the following theorem.

Theorem 8.1 Let $M$ be a $2 n$-dimensional quasitoric manifold. Then we have

$$
N(M) \leq n^{2}+2 n,
$$

with equality only holding for $M=\mathbb{C P}^{n}$.

The proof of this theorem is subdivided into several lemmas. We prove it separately in each dimension. We begin with dimensions $2 n \geq 20$.

Lemma 8.2 Let $M$ be a quasitoric manifold of dimension $2 n \geq 20$ with $M \neq \mathbb{C P}^{n}$. Then we have $N(M) \leq n^{2}+n+1<n^{2}+2 n=N\left(\mathbb{C P}^{n}\right)$.

Proof It was shown by Ku, Mann, Sicks and Su [14, Theorem 1] that if $H^{\alpha}(M ; \mathbb{Q}) \neq 0$ and $M \neq \mathbb{C P}^{n}$, then

$$
N(M) \leq \frac{1}{2} \alpha(\alpha+1)+\frac{1}{2}(2 n-\alpha)(2 n-\alpha+1) .
$$

The statement follows from this result applied in the cases $\alpha=n$ or $\alpha=n-1$.

Now we turn to the low dimensional case $2 n \leq 8$.

Lemma 8.3 Let $M$ be a quasitoric manifold of dimension $2 n, n \leq 4$, and $G$ a compact connected Lie group which acts almost effectively on $M$. Then $\operatorname{dim} G \leq n^{2}+2 n$ and equality only holds for $M=\mathbb{C P}^{n}$ and $\widetilde{G}=\mathrm{SU}(n+1)$. 
Proof Because $M$ has nonzero Euler characteristic, we have rank $G \leq n$. If we have rank $G=n$, it follows from [18, Remark 2.9] that $G$ has a covering group of the form $\widetilde{G}=\prod_{i=1}^{k} \operatorname{SU}\left(l_{i}+1\right) \times T^{l_{0}}$ with $\sum_{i=0}^{k} l_{i}=n$. Therefore we have $\operatorname{dim} G \leq n^{2}+2 n$ with equality holding if and only if $\widetilde{G}=\mathrm{SU}(n+1)$. In the latter case it follows from [18, Corollary 8.9] that $M=\mathbb{C P}^{n}$.

Now assume that $\operatorname{rank} G \leq n-1$. The highest dimensional Lie groups of rank $k$ are:

\begin{tabular}{|c|c|c|c|}
\hline$k$ & $G$ & $\operatorname{dim} G$ & $(k+1)^{2}+2(k+1)$ \\
\hline 1 & $\operatorname{Spin}(3)$ & 3 & 4 \\
2 & $G_{2}$ & 14 & 15 \\
3 & $\operatorname{Spin}(7)$ & 21 & 24 \\
\hline
\end{tabular}

Therefore the statement follows.

Now we turn to the middle dimensions $10 \leq 2 n \leq 18$. Those $2 n$-dimensional simply connected manifolds on which compact connected nonabelian Lie groups of rank $n$ act were classified in [18]. Therefore we first focus on actions of those groups which have a rank which is smaller than $n$.

As a first step we show that if a high-dimensional Lie group acts on a quasitoric manifolds of these dimensions, then its simply connected covering group has a big simple factor which is isomorphic to $\operatorname{Spin}(k)$.

Lemma 8.4 Let $M$ be a manifold of dimension $2 n, 5 \leq n \leq 9$, and $G$ a compact connected Lie group with $\operatorname{rank} G \leq n-1$ and $\operatorname{dim} G \geq n^{2}+2 n$ that acts almost effectively on $M$. Then $G$ has a covering group of the form

$$
\widetilde{G}=\operatorname{Spin}(k) \times G^{\prime},
$$

with $k=9$ if $n=5$ and

$$
k \geq \begin{cases}11 & \text { if } n=6 \\ 12 & \text { if } n=7 \\ 13 & \text { if } n=8 \\ 15 & \text { if } n=9\end{cases}
$$

Proof Let $G / H$ be a principal orbit type of the $G$-action on $M$. Then

$$
\operatorname{dim} G \geq n^{2}+2 n \geq\left(\frac{n}{2}+1\right) \operatorname{dim} M \geq\left(\frac{n}{2}+1\right) \operatorname{dim} G / H .
$$


Because $n / 2+1 \geq 14 / 4$, we may apply [14, Proposition B] with $r=n / 2+1-\epsilon$. Therefore $\widetilde{G}$ is of the form

$$
\begin{aligned}
\operatorname{Spin}(k) \times G^{\prime} & k \geq n+2, \text { or } \\
\operatorname{SU}(k) \times G^{\prime} & k \geq n+1, \text { or } \\
\operatorname{Sp}(k) \times G^{\prime} & k \geq n .
\end{aligned}
$$

Because $\operatorname{rank} G \leq n-1$, the last two cases do not occur.

It remains to prove that the lower bound for $k$ given in the lemma holds. This follows from an inspection of the dimensions of those groups which have $\operatorname{Spin}(k), k \geq n+2$, as a simple factor and rank bounded from above by $n-1$. These groups are listed in the following tables. Here we have omitted those groups which are not isomorphic to $\operatorname{Spin}(k)$ and for which the $\operatorname{Spin}(k)$-factor alone has a dimension greater or equal to $n^{2}+2 n$. If the $\operatorname{Spin}(k)$-factor has a lower dimension, we have only listed those groups which have maximal dimension among those groups which have this $\operatorname{Spin}(k)$-factor. If $n=5$ we have $n^{2}+2 n=35$ and:

\begin{tabular}{|c|c|}
\hline$G$ & $\operatorname{dim} G$ \\
\hline Spin(9) & 36 \\
Spin(8) & 28 \\
$\operatorname{Spin(3)\times Spin(7)~}$ & 24 \\
\hline
\end{tabular}

For $n=6$ we have $n^{2}+2 n=48$ and:

\begin{tabular}{|c|c|}
\hline$G$ & $\operatorname{dim} G$ \\
\hline Spin(11) & 55 \\
Spin(10) & 45 \\
Spin(3) $\times \operatorname{Spin}(9)$ & 39 \\
\hline
\end{tabular}

For $n=7$ we have $n^{2}+2 n=63$ and:

\begin{tabular}{|c|c|}
\hline$G$ & $\operatorname{dim} G$ \\
\hline $\operatorname{Spin}(13)$ & 78 \\
$\operatorname{Spin}(12)$ & 66 \\
$\operatorname{Spin}(3) \times \operatorname{Spin}(11)$ & 58 \\
$G_{2} \times \operatorname{Spin}(9)$ & 50 \\
\hline
\end{tabular}

For $n=8$ we have $n^{2}+2 n=80$ and: 


\begin{tabular}{|c|c|}
\hline$G$ & $\operatorname{dim} G$ \\
\hline $\operatorname{Spin}(15)$ & 105 \\
$\operatorname{Spin}(14)$ & 91 \\
$\operatorname{Spin}(3) \times \operatorname{Spin}(13)$ & 81 \\
$\operatorname{Spin}(3) \times \operatorname{Spin}(12)$ & 69 \\
$G_{2} \times \operatorname{Spin}(11)$ & 69 \\
\hline
\end{tabular}

For $n=9$ we have $n^{2}+2 n=99$ and:

\begin{tabular}{|c|c|}
\hline$G$ & $\operatorname{dim} G$ \\
\hline $\operatorname{Spin}(17)$ & 136 \\
$\operatorname{Spin}(16)$ & 120 \\
$\operatorname{Spin}(15)$ & 105 \\
$\operatorname{Spin}(3) \times \operatorname{Spin}(14)$ & 94 \\
$G_{2} \times \operatorname{Spin}(13)$ & 92 \\
$\operatorname{Spin}(7) \times \operatorname{Spin}(11)$ & 69 \\
\hline
\end{tabular}

Therefore the statement about $k$ follows.

The next step is to identify the identity component of the principal isotropy group of the $\operatorname{Spin}(k)$-action on $M$.

Lemma 8.5 Let $M, G$ as in Lemma 8.4. If $n=5$, then also assume that $\chi(M) \neq 0$. Then the identity component of the principal isotropy group of the $\operatorname{Spin}(k)$-action on $M$ is $\operatorname{Spin}(k-1)$.

Proof If $6 \leq n \leq 9$, one can argue as in the proof of the main lemma of [14, page 135] in Case III.

Therefore assume that $n=5$. Then we have $k=9$. Because $\chi(M) \neq 0$, there is a point $x \in M$ such that $\operatorname{Spin}(9)_{x}$ has maximal rank in $\operatorname{Spin}(9)$. By the classification of maximal rank subgroups of Spin(9) given by Borel and de Siebenthal [4] and the dimension assumption, it follows that $\operatorname{Spin}(9)_{x}^{0}=\operatorname{Spin}(8)$ or $\operatorname{Spin}(9)_{x}^{0}=\operatorname{Spin}(9)$.

If $\operatorname{Spin}(9)_{x}^{0}=\operatorname{Spin}(8)$, then the orbit of $x$ has codimension two in $M$. Because $\operatorname{Spin}(8)$ has no nontrivial 2-dimensional representation, it follows that $\operatorname{Spin}(8)$ is the identity component of a principal isotropy group.

If $\operatorname{Spin}(9)_{x}^{0}=\operatorname{Spin}(9)$, then $T_{x} M$ is a 10 -dimensional representation of $\operatorname{Spin}(9)$. Therefore it is the sum of the standard 9-dimensional representation of $\operatorname{Spin}(9)$ and the trivial one dimensional representation. Hence, the statement follows in this case. 
As a consequence of Lemmas 8.4 and 8.5 we get the following lemma which implies Theorem 8.1 in the remaining dimensions.

Lemma 8.6 Let $M$ be a quasitoric manifold of dimension $2 n, 5 \leq n \leq 9$, and $G$ be a compact connected Lie group which acts almost effectively on $M$.

Then $\operatorname{dim} G \leq n^{2}+2 n$ and equality only holds for $M=\mathbb{C P}^{n}$ and $\widetilde{G}=\mathrm{SU}(n+1)$.

Proof Since $M$ has nonzero Euler characteristic, we have rank $G \leq n$. In the case rank $G=n$, one can argue as in the proof of Lemma 8.3.

Therefore we may assume that $\operatorname{rank} G \leq n-1$. Assume that $\operatorname{dim} G \geq n^{2}+2 n$. By Lemmas 8.4 and 8.5, there is an almost effective action of $\operatorname{Spin}(k)$ on $M$ such that $\operatorname{dim} M / \operatorname{Spin}(k) \leq 4$ and all orbits are acyclic over $\mathbb{Q}$ up to dimension 7 . By the Vietoris-Begle mapping theorem, it follows that

$$
0 \neq H^{6}(M ; \mathbb{Q}) \cong H^{6}(M / \operatorname{Spin}(k) ; \mathbb{Q})=0 .
$$

This is a contradiction.

\section{Appendix A Groups acting on tori}

In this appendix we prove some of the technical details which are needed in the proof of Lemma 2.10.

Lemma A.1 Let $M$ be a free, finitely generated $\mathbb{Z}$-module and $G$ a finite group which acts on $M$. Then there is a $G$-invariant submodule $M^{\prime} \subset M$ such that:

(1) $M^{\prime} \cap M^{G}=\{0\}$.

(2) $\operatorname{rank} M^{\prime}+\operatorname{rank} M^{G}=\operatorname{rank} M$.

Proof Choose a positive definite $G$-invariant metric on $M$. Then the orthogonal complement $M^{\prime}$ of $M^{G}$ is $G$-invariant and $M^{\prime} \cap M^{G}=\{0\}$. Moreover, we have $\operatorname{rank} M^{\prime}+\operatorname{rank} M^{G}=\operatorname{rank} M$.

Lemma A.2 Let $G$ be a finite group, which acts by automorphisms on the torus $T$. Then there are subtori $T_{1}, T_{2} \subset T$ such that:

(1) $T_{1} \subset T^{G}$.

(2) $T_{2}^{G}$ is finite.

(3) $\left\langle T_{1}, T_{2}\right\rangle=T$. 
Proof The action of $G$ on $T$ induces an action of $G$ on the Lie algebra $L T$ of $T$. Let $M$ be the integer lattice in $L T$. Then $M$ is $G$-invariant. Let $M^{\prime}$ and $M^{G}$ as in Lemma A.1 and $T_{2}$ be the subtorus of $T$ corresponding to $M^{\prime} ; T_{1}$ the subtorus of $T$ corresponding to $M^{G}$.

Then we have:

- $T_{1} \subset T^{G}$ because $G$ acts trivially on the Lie algebra of $T_{1}$.

- $T_{2}^{G}$ is finite because of (1) in Lemma A.1.

- $\left\langle T_{1}, T_{2}\right\rangle=T$ because of (1) and (2) in Lemma A.1.

\section{References}

[1] MF Atiyah, R Bott, A Shapiro, Clifford modules, Topology 3 (1964) 3-38 MR0167985

[2] M F Atiyah, I M Singer, The index of elliptic operators, III, Ann. of Math. 87 (1968) 546-604 MR0236952

[3] A Borel, Topics in the homology theory of fibre bundles, Lecture Notes in Mathematics 36, Springer, Berlin (1967) MR0221507

[4] A Borel, J De Siebenthal, Les sous-groupes fermés de rang maximum des groupes de Lie clos, Comment. Math. Helv. 23 (1949) 200-221 MR0032659

[5] V M Buchstaber, T E Panov, Torus actions and their applications in topology and combinatorics, University Lecture Series 24, Amer. Math. Soc. (2002) MR1897064

[6] M W Davis, T Januszkiewicz, Convex polytopes, Coxeter orbifolds and torus actions, Duke Math. J. 62 (1991) 417-451 MR1104531

[7] A Dessai, Spin ${ }^{c}$-manifolds with Pin(2)-action, Math. Ann. 315 (1999) 511-528 MR1731460

[8] A Dessai, Rigidity theorems for Spin $^{c}$-manifolds, Topology 39 (2000) 239-258 MR1722036

[9] A Hattori, Spin ${ }^{c}$-structures and $S^{1}$-actions, Invent. Math. 48 (1978) 7-31 MR508087

[10] A Hattori, T Yoshida, Lifting compact group actions in fiber bundles, Japan. J. Math. 2 (1976) 13-25 MR0461538

[11] V Hauschild, The Euler characteristic as an obstruction to compact Lie group actions, Trans. Amer. Math. Soc. 298 (1986) 549-578 MR860380

[12] F Hirzebruch, P Slodowy, Elliptic genera, involutions, and homogeneous spin manifolds, Geom. Dedicata 35 (1990) 309-343 MR1066570 
[13] M Joswig, Projectivities in simplicial complexes and colorings of simple polytopes, Math. Z. 240 (2002) 243-259 MR1900311

[14] H T Ku, L N Mann, J L Sicks, J C Su, Degree of symmetry of a product manifold, Trans. Amer. Math. Soc. 146 (1969) 133-149 MR0250340

[15] P Orlik, F Raymond, Actions of the torus on 4-manifolds, I, Trans. Amer. Math. Soc. 152 (1970) 531-559 MR0268911

[16] T Petrie, Smooth $S^{1}$ actions on homotopy complex projective spaces and related topics, Bull. Amer. Math. Soc. 78 (1972) 105-153 MR0296970

[17] H Samelson, Notes on Lie algebras, 2nd edition, Springer, New York (1990) MR1056083

[18] M Wiemeler, Torus manifolds with non-abelian symmetries, Trans. Amer. Math. Soc. 364 (2012) 1427-1487 MR2869182

[19] M Wiemeler, Quasitoric manifolds homeomorphic to homogeneous spaces, to appear in Osaka J. Math. 50 (2013) arXiv:1101.0999v2

Karlsruher Institut für Technologie, Institut für Algebra und Geometrie Kaiserstrasse 89-93, D-76133 Karlsruhe, Germany

michael.wiemeler@kit.edu

Received: 18 August 2011 Revised: 22 March 2012 